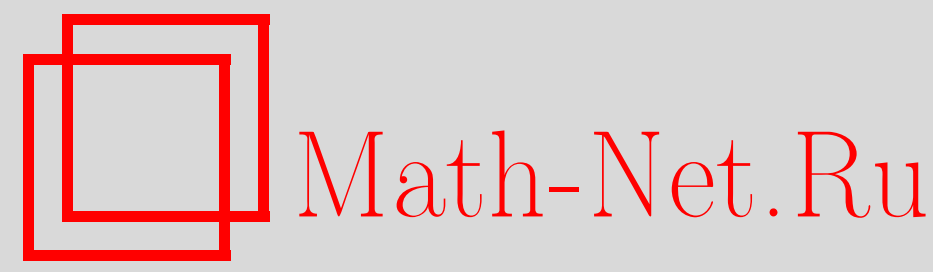

К. И. Чернышов, Об операторе Коши нестационарного линейного дифференциального уравнения с малым параметром при производной, Матем. сб., 2005, том 196, номер 8, 75-118

DOI: https://doi.org/10.4213/sm1407

Использование Общероссийского математического портала Math-Net.Ru подразумевает, что вы прочитали и согласны с пользовательским соглашением

http://www.mathnet.ru/rus/agreement

Параметры загрузки:

IP : 35.174 .16 .151

26 апреля 2023 г., 16:14:49 
УДК 517.98

\author{
К.И. Чернышов
}

\title{
Об операторе Коши нестационарного линейного дифференциального уравнения с малым параметром при производной
}

\begin{abstract}
Предложен алгоритм диагонализации матричного пучка, зависящего от переменной и от параметра, в случаях, когда предельная матрица при всех значениях переменной имеет простой спектр или кратное собственное значение. В алгоритме используется исчерпывающая суперпозиция специальных преобразований подобия. Получены формулы для оператора Коши линейного нестационарного уравнения с мальм параметром при производной и матричньмм пучком при различных степенях вырождения структурной матрицы.

Библиография: 24 названия.
\end{abstract}

\section{§1. Введение}

Пусть $E$ - линейное конечномерное пространство размерности $m, \mathfrak{N}$ - линейное пространство вещественных матриц порядка $m \times m$, действующих в $E$ и непрерьвно зависящих от переменной $t$, изменяющейся на конечном отрезке $[0, T]$, и $\varepsilon-$ мальй параметр, $0<\varepsilon \leqslant \varepsilon_{0}$. Рассмотрим матричный пучок

$$
D(t, \varepsilon)=D_{0}(t)-\sum_{i=1}^{r} \varepsilon^{i} D_{i}(t)
$$

из $\mathfrak{N}$ и задачу Коши для нестационарного линейного дифференциального уравнения (ЛДУ) вида

$$
\varepsilon^{p} \dot{y}=D(t, \varepsilon) y, \quad y(0, \varepsilon)=y_{0} \in E, \quad 0 \leqslant t \leqslant T, \quad 0<\varepsilon \leqslant \varepsilon_{0} .
$$

Для упрощения изложения далее будем считать, что $r=1, p=1$ в (1.1). Тогда соответствуюший оператор Коши уравнения (1.1) является решением задачи

$$
\varepsilon \dot{W}=\left(D_{0}(t)-\varepsilon D_{1}(t)\right) W, \quad W(0, \varepsilon)=I, \quad 0 \leqslant t \leqslant T, \quad 0<\varepsilon \leqslant \varepsilon_{0},
$$

где $I$ - единичная матрица.

Решением задачи (1.2) назовем непрерывно дифференцируемую функцию

$$
W:[0, T] \times\left(0, \varepsilon_{0}\right] \rightarrow \mathfrak{N}
$$

такую, что $\dot{W} \in \mathfrak{N}$ при всех $0 \leqslant t \leqslant T, 0<\varepsilon \leqslant \varepsilon_{0}$, и удовлетворяюшую равенствам $W(0, \varepsilon)=I$ при всех $0<\varepsilon \leqslant \varepsilon_{0}, \varepsilon \dot{W}=\left(D_{0}(t)-\varepsilon D_{1}(t)\right) W$ при всех $0 \leqslant t \leqslant T$, $0<\varepsilon \leqslant \varepsilon_{0}$, где $\varepsilon_{0}$ достаточно мало. 
В настоящей работе предложен алгоритм диагонализации матричного пучка $D_{0}(t)-\varepsilon D_{1}(t)$, на основе которого ведется построение оператора Коши и его асимптотики. С единой точки зрения изучаются ситуации, когда матрица $D_{0}(t)$ (ее называют главной или предельной) имеет при всех $0 \leqslant t \leqslant T$

I) различные собственные значения (простой спектр);

II) $m$-кратное собственное значение (кратньй спектр).

Алгоритм содержит серию различных типов преобразований подобия: $\Phi(t)$, $I+\varepsilon^{\nu} Z_{*}(t, \varepsilon), I+\varepsilon \Omega_{-n}(t), \Lambda(\varepsilon), \Phi^{(1)}(t), I+\varepsilon^{\nu} Y(t, \varepsilon), \nu>0$, вводимых ниже в $\S \S 2,6,8,10,11,14$. Среди них:

а) матрица, столбцами которой служат или собственные векторы, отвечающие различным собственным значениям, или собственный и присоединенные к нему векторы, отвечающие $m$-кратному собственному значению;

b) преобразования, близкие к тождественным, которые возмушены мальм слагаемым, являющимся матрицей или сплетающей, или однодиагональной, или имеющей нулевую диагональ; каждая из матриц является решением соответствующего уравнения с трансформатором (см. $\S 4)$;

c) преобразование срезания Территина;

d) матрица Вандермонда или ее обобщение.

C помощью указанных преобразований осуществляется смена базиса, покоординатная или блочная диагонализация как в главном члене, так и в младших членах пучка, вводится иерархия среди однодиагональных матриц, выделяется главная часть однодиагональной матрицы, играющая решающую роль в перестройке пучка. Некоторые из этих преобразований иног да могут оказаться невостребованньми, и тогда их заменяют матрицей $I$.

В случае простого, или стабильного спектра удается распространить алгоритм диагонализации матричного пучка на часто встречающуюся на практике ситуацию, когда весь пучок состоит из линейного непрерывно обратимого замкнутого симметрического оператора $A(t)$, действующего при каждом $t \in[0, T]$ в сепарабельном гильбертовом пространстве $E$ (см. $\S 7$, п. 7.3).

В случае, когда матрица $D_{0}(t)$ имеет $m$-кратное собственное значение $\lambda(t)$, т.е. когда она подобна матрице $J+\lambda(t) I$, где $J$ - жорданова клетка $m \times m$, отвечающая нулевому собственному значению, в настоящей работе приводятся различные достаточные условия диагонализации матричного пучка в форме ограничений на структурную матрицу $\mathfrak{A}(t)$ (см. (2.4)). Для их описания введем неотрицательные целые числа $n$ и $m_{1}$ такие, что $m_{1}+n=m$, и обозначим

$$
s=n-m_{1}=2 n-m=m-2 m_{1} .
$$

В работе предполагается, что справедливы неравенства $1 \leqslant s \leqslant m$, или $0 \leqslant$ $m_{1} \leqslant(m-1) / 2$, или $(m+1) / 2 \leqslant n \leqslant m$. Одним из основных этапов алгоритма является перестройка пучка путем присоединения к $J$ однодиагональной матриџы $\mathscr{D}_{1-n}$ с единственным ненулевьм при всех $0 \leqslant t \leqslant T$ элементом $\gamma_{1-n}(t)$, pacположенным в $m$-й строке и $\left(m_{1}+1\right)$-м столбце. В результате придем (см. п. 11.2$)$ к предельной матрице $\Gamma(t)$ с собственными значениями $q_{1}(t)$, возмущающими собственное значение $\lambda(t)$. Уравнение $\operatorname{det}\left(\Gamma(t)-q_{1}(t) I\right) \equiv 0$ приводит к разрешаюшему уравнению

$$
q_{1}^{m_{1}}\left(q_{1}^{n}+\gamma_{1-n}(t)\right) \equiv 0
$$


Будем говорить о “невырожденной” структурной матрице, если $m_{1}=0$, и о ее слабом вырождении, когда $m_{1}=1$. В обоих случаях собственные значения матрицы $\Gamma(t)$ попарно различны, однако при $m_{1}=0$ они все ненулевые (случай I)), при $m_{1}=1$ среди собственных значений имеется простой нуль (случай II)). Вместе случаи I) и II) назовем каноническими. Ситуацию, когда $2 \leqslant m_{1} \leqslant(m-1) / 2$, назовем случаем сильного вырождения и подчеркнем, что матрица $\Gamma(t)$ имеет $n$ попарно различных ненулевых собственных значений и одно нулевое $m_{1}$-кратное собственное значение, что приводит к дефиииту решений в полной системе.

Ранее многими авторами изучался эволюционный вариант этой задачи, ставшей классической, т.е. строилась асимптотика решения задачи (1.1). При этом случай простого спектра исследовался в [1] (в координатной форме), в [2], [3], а также в [4], [5] методом регуляризации. Что касается случая кратного спектра, то в [6]-[11] асимптотика решения задачи (1.1) строилась только в канонических случаях, в частности, случай II) исследовался в [9], [10] методом регуляризации, не позволившим отщепить возникающее скалярное ЛДУ. Случай сильного вырождения структурной матрицы в предшествуюшей литературе не рассматривался, в [9], [10] был лишш анонсирован случай $m_{1}=2$. Для полноты изложения упомянем о других способах расшепления линейных систем, приведенных в [12], [13].

Автором в [14]-[16] строилась асимптотика решения задачи (1.1) при сильном вырождении структурной матрищы, однако без использования преобразования подобия $I+\varepsilon^{\nu} Z_{*}(t, \varepsilon), \nu>0$, из $\S 6$, используемого здесь как в случае простого, так и в случае кратного спектра. В [17] велось построение асимптотики решения задачи (1.2) при сильном вырождении структурной матрицы для пучка $D(\varepsilon)$, не зависящего от переменной $t$.

В $\S 7$ приведен алгоритм диагонализации в случае простого спектра, модернизированньй за счет возмущения собственных значений и специального формирования элементов диагональных матриц, что позволяет избегать появления ДУ в качестве условий разрешимости уравнений итерационной процедуры. В $\S 15$ содержится алгоритм диагонализации матричного пучка в случае кратного спектра при $0 \leqslant m_{1} \leqslant(m-1) / 2$. В $\S \S 16,17$ для канонических случаев получено представление оператора Коши в виде

$$
W(t, \varepsilon)=\mathscr{S}(t, \varepsilon) \operatorname{diag}\left(e^{\widetilde{\mu}_{1}(t, \varepsilon)}, \ldots, e^{\widetilde{\mu}_{m}(t, \varepsilon)}\right) \mathscr{S}^{-1}(0, \varepsilon)
$$

где $\mathscr{S}(t, \varepsilon)$ - исчерпьвающая суперпозиция указанных преобразований подобия, скалярные функции $\varepsilon \widetilde{\mu}_{j}(t, \varepsilon)$ зависят от $\varepsilon$ регулярно, причем $\widetilde{\mu}_{i}(t, \varepsilon) \neq \widetilde{\mu}_{j}(t, \varepsilon)$, $i \neq j$, при всех $0 \leqslant t \leqslant T$ и всех $0<\varepsilon \leqslant \varepsilon_{0}$, $\varepsilon_{0}$ достаточно мало, $1 \leqslant i \leqslant m$, $1 \leqslant j \leqslant m$. Формула (1.5) находит применение также в случае простого спектра (при этом набор преобразований подобия, составляющих $\mathscr{S}(t, \varepsilon)$, будет иным) и в случае сильного вырождения, поскольку исходное уравнение удается расшепить на два уравнения в дизъюнктных подпространствах размерности $n, m_{1}$ соответственно. Оператор Коши преобразованного уравнения оказывается представимьп в виде суммы двух слагаемых, каждое из которых действует в своем подпространстве. Одно из них по форме не отличается от (1.5), и именно этим объясняется необходимость обращения к случаю простого спектра. Второе окончательно формируется, когда в соответствуюшем $m_{1}$-мерном подпространстве имеет место один 
из канонических случаев (см. $\S 18)$, иначе за первой редукцией задачи должна последовать вторая и т.д. Исследование завершается, когда все возмушения $\widetilde{\mu}_{j}(t, \varepsilon)$, $1 \leqslant j \leqslant m$, становятся попарно различными.

Предложенный в настоящей работе метод построения оператора Коши и его асимптотики применим к различньм типам уравнений с малым параметром при производной, с большим параметром в правой части, с медленно меняющимися коэффициентами.

\section{§2. Матрица подобия $\Phi(t)$}

\section{1. Случай простого спектра.}

ОПРЕДЕЛЕНИЕ 2.1. Будем Говорить, что матрица порядка $m \times m$ имеет $n p o c-$ той спектр на отрезке $[0, T]$, если ее собственные значения при всех $0 \leqslant t \leqslant T$ удовлетворяют соотношениям

$$
\lambda_{i}(t) \neq \lambda_{j}(t), \quad i \neq j ; \quad \lambda_{i}(t) \neq 0, \quad 1 \leqslant i \leqslant m, \quad 1 \leqslant j \leqslant m .
$$

ЗАмЕчАнИЕ 2.1. В [2; гл. $5, \S 2$, п. 1.3] эти соотношения названы условием отсутствия точек поворота при $t \in[0, T]$.

Пусть матрица $D_{0}(t)$ порядка $m \times m$ имеет простой спектр на отрезке $[0, T]$. Обозначим через $\varphi_{r}(t)$ ее собственный вектор, отвечающий собственному значению $\lambda_{r}(t), 1 \leqslant r \leqslant m$, и выберем систему векторов $\left\{\varphi_{r}(t)\right\}, 1 \leqslant r \leqslant m$, в качестве базиса пространства $E$. Обозначим через $\Phi(t)$ обратимую матрицу, столбцами которой служат координаты векторов $\varphi_{1}(t), \ldots, \varphi_{m}(t)$ в ортонормированном базисе $\left\{e_{r}\right\}$, где $e_{r}$ - вектор, единственным ненулевым элементом которого является единица, стоящая на $r$-м месте, $1 \leqslant r \leqslant m$. Применение преобразования $\Phi(t)$ позволяет снабдить пространство $E$ базисом $\left\{e_{r}\right\}, 1 \leqslant r \leqslant m$, и рассматривать матрицы из $\mathfrak{N}$ при каждом $t, 0 \leqslant t \leqslant T$, в алгебре End $E$.

Здесь и ниже через $\operatorname{Hom}(E, F)$ обозначается банахово пространство линейных ограниченных операторов, действующих из $E$ в $F$, а через End $E$ - банахова алгебра линейных ограниченных операторов (эндоморфизмов), действующих в пространстве $E$.

Теперь от матрицы $D_{0}(t)$, являющейся диагональной в базисе $\left\{\varphi_{r}(t)\right\}$, можно перейти к диагональной матрице $Q(t)=\operatorname{diag}\left(\lambda_{1}(t), \ldots, \lambda_{m}(t)\right)$ в базисе $\left\{e_{r}\right\}$. Через $\mathfrak{H}(t)$ обозначим матрищу, столбцами которой являются координаты разложений векторов $\dot{\varphi}_{1}(t), \ldots, \dot{\varphi}_{m}(t)$ в базисе $\left\{\varphi_{r}(t)\right\}$. Тогда

$$
\mathfrak{H}(t)=\Phi^{-1}(t) \dot{\Phi}(t) .
$$

$$
\begin{aligned}
\text { Поскольку } 0=\frac{d}{d t}\left(\Phi^{-1} \Phi\right) & =\dot{\Phi}^{-1} \Phi+\mathfrak{H}, \text { то } \\
\mathfrak{H} & =-\dot{\Phi}^{-1} \Phi, \quad \dot{\Phi}^{-1}=-\mathfrak{H} \Phi^{-1} .
\end{aligned}
$$

Равенства $\varphi_{r}(t)=\Phi(t) e_{r},\left(D_{0}(t)-\lambda_{r}(t) I\right) \varphi_{r}(t)=0,1 \leqslant r \leqslant m$, влекут соотношение

$$
\Phi^{-1}(t) D_{0}(t) \Phi(t)=Q(t) .
$$

В дальнейшем важную роль играет матрица

$$
\mathfrak{A}(t)=\mathfrak{H}(t)+\Phi^{-1}(t) D_{1}(t) \Phi(t),
$$

которую, следуя [9], [10], назовем структурной. 
ЗАмечАниЕ 2.2. Структурная матрица возникает и при $D_{1}(t) \equiv 0$.

\section{2. Случай кратного спектра.}

ОПРЕДЕЛЕНИЕ 2.2. Будем говорить, что матрица порядка $m \times m$ uмeет $m$-кратное собственное значение $\lambda(t)$ (кратный спектр) на отрезке $[0, T]$, если при всех $0 \leqslant t \leqslant T$ выполнены соотношения

$$
\left(D_{0}(t)-\lambda(t) I\right) \varphi_{1}(t)=0, \quad\left(D_{0}(t)-\lambda(t) I\right) \varphi_{r}(t)=\varphi_{r-1}(t), \quad 2 \leqslant r \leqslant m,
$$

причем $\varphi_{m}(t) \notin \operatorname{Im}\left(D_{0}(t)-\lambda(t) I\right)$.

Пусть матрица $D_{0}(t)$ имеет $m$-кратное собственное значение $\lambda(t)$ на отрезке $[0, T]$. Систему векторов $\left\{\varphi_{r}(t)\right\}, 1 \leqslant r \leqslant m$, можно выбрать в качестве базиса пространства $E$. Составим обратимую матрицу $\Phi(t)$, столбцами которой служат координаты векторов $\varphi_{1}(t), \ldots, \varphi_{m}(t)$ в базисе $\left\{e_{r}\right\}, 1 \leqslant r \leqslant m$, а также матрицу $\mathfrak{H}(t)$, столбцами которой являются координаты векторов $\dot{\varphi}_{1}(t), \ldots, \dot{\varphi}_{m}(t)$ в базисе $\left\{\varphi_{r}(t)\right\}$, удовлетворяющую равенствам $(2.2),(2.4)$. Кроме того, поскольку $\Phi^{-1}(t)\left(D_{0}(t)-\lambda(t) I\right) \Phi(t)=J$, то здесь удается от матрицы $D_{0}(t)$, которая подобна матрице $J+\lambda(t) I$, перейти к $J$, и, значит,

$$
\Phi^{-1}(t)((D(t, \varepsilon)-\lambda(t) I) \Phi(t)-\varepsilon \dot{\Phi}(t))=J-\varepsilon \mathfrak{A}(t) .
$$

\section{§ 3. Структура пространства $\mathfrak{N}$. Однодиагональные матрицы}

3.1. Базис в пространстве $\mathfrak{N}$. В $m^{2}$-мерном пространстве $\mathfrak{N}$ введем базис с помошью $m^{2}$ матриц $V_{i-m}^{r, i-1-r}, V_{m-i}^{r, i-1-r}$, у которых единственный ненулевой элемент равен 1 и расположен в $(m-i+r+1)$-й строке, $(r+1)$-м столбце или в $(r+1)$-й строке, $(m-i+r+1)$-м столбце соответственно, $0 \leqslant r \leqslant i-1$, $1 \leqslant i \leqslant m$. Единица расположена на линии, параллельной диагонали и состолшей из $i$ элементов, причем на этой линии выше единицы находятся $r$ нулей, а ниже $i-1-r$ нулей (об этом свидетельствуют верхние индексы). В первой группематриц указанная линия лежит не вьше диагонали, во второй - не ниже ее.

Непосредственной проверкой устанавливается, что при умножении базисных элементов нижний индекс произведения равен сумме нижних индексов сомножителей, т.е. имеет место логарифмический закон. Этот закон справедлив также и для их коммутатора. Здесь и далее коммутатор матриц $F, G$, равный $F G-G F$, обозначается через $[F, G]$.

3.2. Однодиагональные матрицы. Обозначим элемент матрицы $X(t)$ из $\mathfrak{N}$, стоящий в $r$-й строке, $l$-м столбце, через $x_{r, l}(t)$ и представим $X(t)$ в виде суммы $o \partial$ нодиагональных матрии $X_{i}(t), 1-m \leqslant i \leqslant m-1$. Элементами $X_{i}(t)$ являются $x_{r, l}(t)$, для которых $l-r=i$. При $1-m \leqslant i \leqslant 0-$ это $x_{1-i, 1}(t), \ldots, x_{m, m+i}(t)$, при $0 \leqslant i \leqslant m-1$ - это $x_{1, i+1}(t), \ldots, x_{m-i, m}(t)$. Ненулевые элементы матрицы $X_{i}(t)$ занимают линию, параллельную диагонали с номером, отсчитываемым снизу вверх от $1-m$ до $m-1$. С помошью базисных элементов в $\mathfrak{N}$ находим, что

$$
\begin{array}{ll}
X_{i}(t)=\sum_{r=1}^{m+i} x_{r-i, r}(t) V_{i}^{r-1, m+i-r}, & 1-m \leqslant i \leqslant 0, \\
X_{i}(t)=\sum_{r=1}^{m-i} x_{r, i+r}(t) V_{i}^{r-1, m-i-r}, & 0 \leqslant i \leqslant m-1 .
\end{array}
$$


Теперь действия с однодиагональными матрицами $X_{i}(t)$ сводятся к действиям с базисными элементами. Используя логарифмический закон, заключаем, что нижний индекс произведения двух однодиагональных матриц или их коммутатора равен сумме нижних индексов сомножителей, причем суммарный нижний индекс ненулевой матрицы принимает значения от $1-m$ до $m-1$.

Условимся обозначать сумму элементов однодиагональной матрицы $X_{i}(t)$ через $\gamma_{i}^{X}(t)$ при всех $1-m \leqslant i \leqslant m-1$.

3.3. Прямые разложения пространства $\mathfrak{N}$. Следуя схеме из [18], используюшей идеи статьи [19], введем в $\mathfrak{N}$ при каждом $t, 0 \leqslant t \leqslant T$, скалярное произведение

$$
(A(t), B(t))=\operatorname{Tr}\left(B^{T}(t) A(t)\right)
$$

где знак “ $T$ ” означает транспонирование. Норму в $\mathfrak{N}$ при каждом $t, 0 \leqslant t \leqslant T$, зададим формулой

$$
\|A(t)\|=\sqrt{\sum_{r=1}^{m} \sum_{j=1}^{m} a_{j, r}^{2}(t)}, \quad 0 \leqslant t \leqslant T .
$$

Всюду в дальнейшем будем использовать обозначение $A(\varepsilon)=O\left(\varepsilon^{\alpha}\right)$, если матрица $A(\varepsilon)$ из алгебры End $E$ подчиняется оценке $\|A(\varepsilon)\| \leqslant M \varepsilon^{\alpha}$ для всех $0<$ $\varepsilon \leqslant \varepsilon_{0}$, где число $M$ не зависит от $\varepsilon$ и $\varepsilon_{0}$ достаточно мало.

Введем в $\mathfrak{N}$ три подпространства $N, N^{\prime}, M$.

ОПРЕДЕЛЕНИЕ 3.1. Матрица $B(t) \in N^{\prime}$, если ее ненулевые элементы занимают линии, параллельные диагонали с номерами от $1-m$ до 0 , причем на каж дой линии элементы одинаковы.

ОПРЕдЕлЕНиЕ 3.2. Матрица $C(t) \in N$, если ее ненулевые элементы занимают линии, параллельные диагонали с номерами от 0 до $m-1$, причем на каж дой линии элементы одинаковы.

ОПРЕДЕЛЕНИЕ 3.3. Матрица $\Pi(t) \in M$, если ее ненулевые элементы занимают только $m$-ю строку.

Отметим, что матрицы из $N, N^{\prime}, M$ содержат $m$ параметров.

Далее, введем еще два подпространства $F, \mathscr{U}$ в $\mathfrak{N}$.

ОПРЕДЕЛЕНИЕ 3.4. Матрица $\Gamma(t) \in F$, если $(\Gamma(t), B(t))=0$ для любой матрицы $B(t) \in N^{\prime}$ и всех $0 \leqslant t \leqslant T$.

ОПРЕДЕЛЕНИЕ 3.5. Матрица $U(t) \in \mathscr{U}$, если $(U(t), C(t))=0$ для любой матрицы $C(t) \in N$ и всех $0 \leqslant t \leqslant T$.

Справедливы следующие утверждения.

Лемма 3.1. Матрица $\Gamma(t) \in F \Longleftrightarrow \gamma_{i}^{\Gamma}(t) \equiv 0, \quad 1-m \leqslant i \leqslant 0,0 \leqslant t \leqslant T$.

Лемма 3.2. Матрица $U(t) \in \mathscr{U} \Longleftrightarrow \gamma_{i}^{U}(t) \equiv 0,0 \leqslant i \leqslant m-1,0 \leqslant t \leqslant T$. 
У тверждения лемм $3.1,3.2$ проверяются непосредственно с учетом определения скалярного произведения в $\mathfrak{N}$. С помощью этих лемм устанавливается, что имеют место прямые разложения вида

$$
\mathfrak{N}=N^{\prime} \stackrel{\perp}{\oplus} F, \quad \mathfrak{N}=N \stackrel{\perp}{\oplus} \mathscr{U}, \quad \mathfrak{N}=M \oplus F .
$$

Обозначим через $J$ жорданову клетку $m \times m$, отвечающую нулевому собственному значению, а через $J^{\prime}$ сопряженную к $J$ матрицу.

Лемма 3.3. С матрицами $J, J^{\prime}$ коммутируют матрицьи из $N, N^{\prime}$ соответственно и только они.

ЗАмечАниЕ 3.1. В [20] фигурирует разложение $\mathfrak{N}=M \oplus F$ пространства $\mathfrak{N}$ в прямую сумму подпространств, одно из которых $(F)$ характеризует интегрируемую (в определенном смысле) часть некоторого оператора, имеющего на другом подпространстве $(M)$ ограниченную высоту.

\section{§4. Уравнения с трансформатором}

4.1. Уравнения $T_{Q, J_{1}} X=Y, T_{J_{1}, Q} X=Y$. Пусть даны матрицы $L(t), M(t)$, $Y(t)$ такие, что $L(t) \in \operatorname{End} \mathbb{R}^{l}, M(t) \in \operatorname{End} \mathbb{R}^{k}, Y \in \operatorname{Hom}\left(\mathbb{R}^{k}, \mathbb{R}^{l}\right)$ при каждом $0 \leqslant t \leqslant T$.

Как и в $\left[21 ;\right.$ гл. I, $\S 3$, п. 1], введем в пространстве $\operatorname{Hom}\left(\mathbb{R}^{k}, \mathbb{R}^{l}\right)$ линейные операторы $L_{l}(t)$ и $M_{r}(t)$, порождаемые умножением оператора $X(t) \in \operatorname{Hom}\left(\mathbb{R}^{k}, \mathbb{R}^{l}\right)$ на оператор $L(t)$ слева и оператор $M(t)$ справа:

$$
L_{l}(t) X(t)=L(t) X(t), \quad M_{r}(t) X(t)=X(t) M(t), \quad X(t) \in \operatorname{Hom}\left(\mathbb{R}^{k}, \mathbb{R}^{l}\right) .
$$

Следуя [21], назовем оператор

$$
T_{L, M}(t)=L_{l}(t)-M_{r}(t), \quad T_{L, M}(t) X(t)=L(t) X(t)-X(t) M(t),
$$

действующий при каждом $t \in[0, T]$ в $\operatorname{Hom}\left(\mathbb{R}^{k}, \mathbb{R}^{l}\right)$, трансформатором Ляпунова. Известно, что условия разрешимости и формула решения уравнения

$$
T_{L, M}(t) X(t)=Y(t)
$$

существенно зависят от взаимного расположения спектров матриц $L(t), M(t)$. Если их спектры не пересекаются, то трансформатор $T_{L, M}(t)$ обратим, т.е. уравнение

$$
L(t) X(t)-X(t) M(t)=Y(t)
$$

имеет единственное решение $X(t)$ при любом $Y(t) \in \operatorname{Hom}\left(\mathbb{R}^{k}, \mathbb{R}^{l}\right)$.

Пусть $J_{1}$ - жорданова клетка $m_{1} \times m_{1}$, отвечающая нулевому собственному значению, и $Q(t)=\operatorname{diag}\left(q_{1}(t), \ldots, q_{n}(t)\right)$ - матрица с ненулевьми попарно различньми собственными значениями при всех $0 \leqslant t \leqslant T$. Поскольку матрица $Q(t)$ обратима при всех $0 \leqslant t \leqslant T$, а $J_{1}$ нильпотентна с индексом $m_{1}$, т.е. $J_{1}^{m_{1}}=0$, то 
равенство $Q X-X J_{1}=Y$ можно представить в форме $X=Q^{-1}\left(Y+X J_{1}\right)$. После $m_{1}$-кратного применения последнего равенства приходим к соотношению

$$
\begin{aligned}
X & =Q^{-1}\left(Y+X J_{1}\right)=Q^{-1} Y+Q^{-1}\left(Q^{-1}\left(Y+X J_{1}\right)\right) J_{1}=\cdots \\
& =Q^{-1} Y+\left(Q^{-1}\right)^{2} Y J_{1}+\left(Q^{-1}\right)^{3} Y J_{1}^{2}+\cdots+\left(Q^{-1}\right)^{m_{1}} Y J_{1}^{m_{1}-1},
\end{aligned}
$$

даюшему формулу обратного оператора к трансформатору $T_{Q(t), J_{1}}$ :

$$
T_{Q(t), J_{1}}^{-1} Y(t)=\sum_{i=0}^{m_{1}-1}\left(Q^{-1}(t)\right)^{i+1} Y(t) J_{1}^{i} .
$$

По аналогии запишем равенство $J_{1} X-X Q=Y$ в форме $X=\left(-Y+J_{1} X\right) Q^{-1}$ и используем его $m_{1}$ раз, получим, что

$$
X=-Y Q^{-1}-J_{1} Y\left(Q^{-1}\right)^{2}-J_{1}^{2} Y\left(Q^{-1}\right)^{3}-\cdots-J_{1}^{m_{1}-1} Y\left(Q^{-1}\right)^{m_{1}} .
$$

Отсюда вытекает формула обратного оператора к трансформатору $T_{J_{1}, Q(t)}$ :

$$
T_{J_{1}, Q(t)}^{-1} Y(t)=-\sum_{i=0}^{m_{1}-1} J_{1}^{i} Y(t)\left(Q^{-1}(t)\right)^{i+1} .
$$

Равенства (4.1), (4.2) влекут следуюшие утверждения.

Лемма 4.1. Уравнение $T_{Q(t), J_{1}} X=Q(t) X-X J_{1}=Y(t)$ разрешимо для любой матрицы $Y(t) \in \operatorname{Hom}\left(\mathbb{R}^{m_{1}}, \mathbb{R}^{n}\right)$. Элементы $x_{r, j}(t)$ матрицы $X(t) \in$ $\operatorname{Hom}\left(\mathbb{R}^{m_{1}}, \mathbb{R}^{n}\right)$ находятся единственным образом и характеризуются равенствами

$$
x_{j, r}(t)=\sum_{i=1}^{r} q_{j}^{r-1-i}(t) y_{j, i}(t), \quad 1 \leqslant j \leqslant n, \quad 1 \leqslant r \leqslant m_{1}, \quad 0 \leqslant t \leqslant T .
$$

Лемма 4.2. Уравнение $T_{J_{1}, Q(t)} X=J_{1} X-X Q(t)=Y(t)$ разрешимо для любой матрицы $Y(t) \in \operatorname{Hom}\left(\mathbb{R}^{n}, \mathbb{R}^{m_{1}}\right)$. Элементы $x_{r, j}(t)$ матрицы $X(t) \in$ $\operatorname{Hom}\left(\mathbb{R}^{n}, \mathbb{R}^{m_{1}}\right)$ находятся единственныц образом и характеризуются равенствами

$$
x_{r, j}(t)=-\sum_{i=r}^{m_{1}} q_{j}^{r-1-i}(t) y_{i, j}(t), \quad 1 \leqslant r \leqslant m_{1}, \quad 1 \leqslant j \leqslant n, \quad 0 \leqslant t \leqslant T .
$$

4.2. Уравнение с трансформатором $K_{0}$. Введем трансформатор $K_{0}(t)=$ $T_{Q(t), Q(t)}=[Q(t), \cdot]$, действующий при каждом $0 \leqslant t \leqslant T$ в End $\mathbb{R}^{n}$, где $Q(t)=$ $\operatorname{diag}\left(q_{1}(t), \ldots, q_{n}(t)\right)$ - матрица с ненулевыми попарно различными собственными значениями при всех $0 \leqslant t \leqslant T$. Так как $\operatorname{Ker} K_{0}(t)$ - это множество всех диагональных матриц, то уравнение $Q(t) X-X Q(t)=Y(t)$ не является разрешимым для любой матрицы $Y(t) \in$ End $\mathbb{R}^{n}$. Проверяется, что $\operatorname{Im} K_{0}(t)$ состоит из всевозможных матриц с нулевой диагональю. 
Пусть $A(t)=\left(a_{k, l}(t)\right), k, l=1,2, \ldots, m,-$ произвольная матрица из $\mathfrak{N}$. Здесь и в дальнейшем будем обозначать

$$
A_{0}(t)=\operatorname{diag}\left(a_{1,1}(t), \ldots, a_{m, m}(t)\right), \quad A_{*}(t)=A(t)-A_{0}(t) .
$$

В соответствии с представлением $A(t)=A_{0}(t)+A_{*}(t)$ имеем разбиение единицы $I=I_{0}+I_{*}$, где $A_{0}(t)=I_{0} A(t), A_{*}(t)=I_{*} A(t)$. Очевидно, что $I_{0}^{2}=I_{0}$, т.е. $I_{0}, I_{*}-$ проекторы.

Лемма 4.3. Уравнение $K_{0}(t) X(t)=Y(t)$ әквивалентно системе

$$
\left\{\begin{array}{l}
I_{0} Y(t) \equiv 0 \\
X(t)=I_{0} X(t)+\widehat{K}_{0}^{-1}(t) I_{*} Y(t)
\end{array}\right.
$$

əде $\widehat{K}_{0}(t)=K_{0}(t) \uparrow \operatorname{Im} K_{0}(t)$.

Проверяется, что если $Y(t)=\left(y_{i j}(t)\right)$, то $\widehat{K}_{0}^{-1}(t) I_{*} Y(t)=\left(y_{i j}(t) /\left(q_{i}(t)-q_{j}(t)\right)\right)$, $i \neq j$.

ЛЕмма 4.4. Уравнение $Q(t) X-X Q(t)=Y(t)$ разрешимо точно тогда, когда $y_{i, i}(t) \equiv 0,1 \leqslant i \leqslant n$. При вьгполнении этих условий существует единственная матрица $X(t)=X_{*}(t)$, әлементы $x_{r, j}(t)$ которой характеризуются равенствами

$$
\begin{gathered}
x_{r, r}(t) \equiv 0, \quad x_{r, j}(t)=\frac{y_{r, j}(t)}{q_{r}(t)-q_{j}(t)}, \quad r \neq j, \\
1 \leqslant r \leqslant n, \quad 1 \leqslant j \leqslant n, \quad 0 \leqslant t \leqslant T .
\end{gathered}
$$

4.3. Уравнение с трансформатором $K_{1}$. Введем трансформатор $K_{1}=$ $T_{J, J}=[J, \cdot]$, действуюший в $\mathfrak{N}$. Из леммы 3.3 вытекает, что $K_{1} C(t)=0$ для любой матрицы $C(t) \in N$, следовательно, $\operatorname{Ker} K_{1}=N$. Покажем, что

$$
\operatorname{Im} K_{1}=F \text {. }
$$

Возьмем произвольную матрицу $\Gamma(t) \in F$ и построим $U(t) \in \mathscr{U}$ такую, что $K_{1} U(t)=\Gamma(t)$. Непосредственньм образом проверяется, что если $\gamma_{i}^{\Gamma}(t) \equiv 0$, $1-m \leqslant i \leqslant 0$, то элементы матрицы $U(t)$ с $\gamma_{i}^{U}(t) \equiv 0,0 \leqslant i \leqslant m-1$, находятся единственным образом. Итак, справедливы разложения пространства $\mathfrak{N}$ в прямые суммы вида

$$
\mathfrak{N}=\operatorname{Ker} K_{1} \stackrel{\perp}{\oplus} \mathscr{U}, \quad \mathfrak{N}=N^{\prime} \stackrel{\perp}{\oplus} \operatorname{Im} K_{1}, \quad \mathfrak{N}=M \oplus \operatorname{Im} K_{1},
$$

причем оба подпространства $N^{\prime}, M$ претендуют на роль подпространства Coker $K_{1}$. Из двух разложений, содержащих $\operatorname{Im} K_{1}$, для наших целей предпочтительнее последнее, поскольку в матрицах из $M$ имеется не только наименее возможное число независимых параметров, но и наименьшее число ненулевых элементов, расположенных в последней $m$-й строке. Таким образом, ниже будут использоваться прямые разложения

$$
\mathfrak{N}=\operatorname{Ker} K_{1} \stackrel{\perp}{\oplus} \mathscr{U}, \quad \mathfrak{N}=M \oplus \operatorname{Im} K_{1} \quad\left(M=\operatorname{Coker} K_{1}\right)
$$

и соответствующие разбиения единиц

$$
I=(I-Q)+Q, \quad I=(I-P)+P .
$$


ЛЕмма 4.5. Уравнение $K_{1} X(t)=Y(t)$ әквивалентно системе

$$
\left\{\begin{array}{l}
(I-P) Y(t)=0 \\
X(t)=(I-Q) X(t)+\widehat{K}_{1}^{-1} P Y(t)
\end{array}\right.
$$

əде $\widehat{K}_{1}=K_{1} \uparrow \mathscr{U}$.

Выясним, как действует проектор $I-P$ из $\mathfrak{N}$ на $M$. С этой целью возьмем произвольную матрицу $Y(t)$ из $\mathfrak{N}$ и представим ее в виде

$$
Y(t)=\Pi(t)+F(t), \quad \Pi(t) \in M, \quad F(t) \in \operatorname{Im} K_{1} .
$$

Так как $(I-P) Y(t)=\Pi(t)=Y(t)-F(t)$, а элементы $F(t)$ удовлетворяют соотношениям $\gamma_{i}^{F}(t) \equiv 0,1-m \leqslant i \leqslant 0$, то, отнимая и добавляя $\gamma_{i}^{Y}(t)$ в $m$-й строке $i$-й линии, $1-m \leqslant i \leqslant 0$, приходим к выводу: $(I-P) Y(t)-$ матрица $m \times m$, ненулевые элементы которой занимают $m$-ю строку и равны $\gamma_{1-m}^{Y}(t), \gamma_{2-m}^{Y}(t), \ldots, \gamma_{-1}^{Y}(t), \gamma_{0}^{Y}(t)$

Теперь можно переформулировать лемму 4.5 следующим образом.

ЛЕмма 4.6. Уравнение $K_{1} X(t)=Y(t)$ разрешимо точно тогда, когда $\gamma_{i}^{Y}(t) \equiv 0,1-m \leqslant i \leqslant 0$. При выполнении этих условий существует единственное решение $\widehat{X}(t)$ из $\mathscr{U}$, обладающее свойством $\gamma_{i}^{\widehat{X}}(t) \equiv 0,0 \leqslant i \leqslant m-1$.

ПримеР 4.1. Пусть $Y(t)=U_{1-n}^{(0)}(t), 2 \leqslant n \leqslant m-1$, причем $\gamma_{1-n}^{(0)}(t) \equiv 0$, где $\gamma_{1-n}^{(0)}(t)$ - сумма элементов матрицы $U_{1-n}^{(0)}(t)$. Так как условие разрешимости из леммы 4.6 выполнено, то, обозначив элементы матрицы $U_{1-n}^{(0)}(t)$ через $\sigma_{r+n-1, r}^{(0)}(t)$, $1 \leqslant r \leqslant m_{1}+1$, и заметив, что

$$
\sigma_{m, m_{1}+1}^{(0)}(t) \equiv-\sum_{r=1}^{m_{1}} \sigma_{n-1+r, r}^{(0)}(t),
$$

найдем решение $\widehat{X}(t)$ из $\mathscr{U}$. Проверяется, что $\widehat{X}(t)=\Omega_{-n}(t)=\left(\omega_{n+r, r}(t)\right)$, где

$$
\omega_{n+r, r}(t)=\sum_{l=1}^{r} \sigma_{n-1+l, l}^{(0)}(t), \quad 1 \leqslant r \leqslant m_{1} .
$$

\section{§5. Операторы Коши некоторых вспомогательных уравнений в случае простого спектра предельной матрицы}

5.1. Дифференциальные уравнения с диагональным пучком. Введем диагональные матрицы

$$
\begin{gathered}
Q(t)=\operatorname{diag}\left(\lambda_{1}(t), \ldots, \lambda_{m}(t)\right), \\
Q_{r}(t)=\operatorname{diag}\left(q_{r, 1}(t), \ldots, q_{r, m}(t)\right), \quad r \in \mathbb{N}, \quad F(t, \varepsilon)=\sum_{r=1}^{\infty} \varepsilon^{r-1} Q_{r}(t) .
\end{gathered}
$$


ПРЕДПОЛОЖЕНИЕ 5.1. Матрица $Q(t)$ имеет простой спектр на $[0, T]$.

Рассмотрим уравнение

$$
\varepsilon \dot{W}=(Q(t)-\varepsilon F(t, \varepsilon)) W, \quad 0 \leqslant t \leqslant T .
$$

Лемма 5.1. Пусть выполнено предположение 5.1. Тогда решением уравнения (5.3) с диагональныц матричныцм пучком

$$
Q(t)-\varepsilon Q_{1}(t)-\varepsilon^{2} Q_{2}(t)-\varepsilon^{3} \cdots
$$

является матричная функция вида

$$
W(t, \varepsilon)=\operatorname{diag}\left(e^{\mu_{1}(t, \varepsilon)}, \ldots, e^{\mu_{m}(t, \varepsilon)}\right) C(\varepsilon),
$$

где матрица $C(\varepsilon)$ произвольна и

$$
\mu_{i}(t, \varepsilon)=\varepsilon^{-1} \int_{0}^{t}\left(\lambda_{i}(\tau)-\sum_{r=1}^{\infty} \varepsilon^{r} q_{r, i}(\tau)\right) d \tau, \quad 1 \leqslant i \leqslant m .
$$

СлЕДСТВИЕ 5.1. Если выполнено предположение 5.1 и в уравнении (5.3) диагональные матрицы $Q(t), Q_{r}(t), r \in \mathbb{N}$, постояннье, то скалярные функции $\mu_{i}(t, \varepsilon)$ определяются равенствами

$$
\mu_{i}(t, \varepsilon)=\frac{t}{\varepsilon}\left(\lambda_{i}-\sum_{r=1}^{\infty} \varepsilon^{r} q_{r, i}\right), \quad 1 \leqslant i \leqslant m .
$$

Рассмотрим задачу Коши $W(0, \varepsilon)=I$ для уравнения (5.3), тогда ее решение является оператором Коши. Справедлива

ЛЕмма 5.2. Если выполнено предположение 5.1, то оператор Коши уравнения (5.3) имеет вид

$$
W_{0}(t, \varepsilon)=\operatorname{diag}\left(e^{\mu_{1}(t, \varepsilon)}, \ldots, e^{\mu_{m}(t, \varepsilon)}\right),
$$

әде скалярные функиии $\mu_{i}(t, \varepsilon)$ определяются соотношениями (5.5).

СлЕДСТВИЕ 5.2. Если выполнено предположение 5.1, то оператором Коии укороченного уравнения

$$
\varepsilon \dot{W}=\left(Q(t)-\varepsilon Q_{1}(t)\right) W
$$

является матричная функиия (5.7) со скалярными функииями $\mu_{i}(t, \varepsilon)$ ви$\partial a(5.5)$ nрu $q_{r, i}(\tau) \equiv 0, r \geqslant 2,1 \leqslant i \leqslant m$.

Рассмотрим задачу Коши $\mathscr{W}(0, \varepsilon)=I$ для уравнения

$$
\varepsilon^{\dot{W}}=-\mathscr{W}(Q(t)-\varepsilon F(t, \varepsilon)), \quad 0 \leqslant t \leqslant T,
$$

союзного по отношению к (5.3). 
ЛЕмма 5.3. Если выполнено предположсение 5.1, то оператор Коши уравнения (5.8) имеет вид

$$
\mathscr{W}_{0}(t, \varepsilon)=\operatorname{diag}\left(e^{-\mu_{1}(t, \varepsilon)}, \ldots, e^{-\mu_{m}(t, \varepsilon)}\right),
$$

где скалярные функции $\mu_{i}(t, \varepsilon)$ определяются соотношениями (5.5).

5.2. Дифференциальное уравнение с линейным пучком. Пусть выполнено предположение 5.1. Обратимся к уравнению

$$
\varepsilon \dot{W}=(Q(t)-\varepsilon \mathfrak{A}(t)) W, \quad 0 \leqslant t \leqslant T,
$$

с матрищей $Q(t)$ из $(5.1)$, произвольной матрицей $\mathfrak{A}(t)$ и построим оператор Коши для него. Представим $\mathfrak{A}(t)=\left(\sigma_{i, j}(t)\right)$ по формуле $(4.3)$ в виде суммы ее диагонали $Q_{1}(t)=\mathfrak{A}_{0}(t)$ и матрищы $\mathfrak{A}_{*}(t)$ с нулевой диагональю. Запишем уравнение $(5.10)$ в форме

$$
\varepsilon \dot{W}=\left(Q(t)-\varepsilon Q_{1}(t)\right) W-\varepsilon \mathfrak{A}_{*}(t) W, \quad 0 \leqslant t \leqslant T,
$$

и воспользуемся методом вариации. Тогда решение уравнения (5.10) согласно следствию 5.2 представимо в виде

$$
W(t, \varepsilon)=W_{0}(t, \varepsilon) C(t, \varepsilon),
$$

где

$$
\mu_{i}(t, \varepsilon)=\varepsilon^{-1} \int_{0}^{t}\left(\lambda_{i}(\tau)-\varepsilon \sigma_{i, i}(\tau)\right) d \tau, \quad 1 \leqslant i \leqslant m
$$

и матричная функция $C(t, \varepsilon)$ с условием $C(0, \varepsilon)=I$ подлежит определению. Подставляя (5.10) в (5.11) и замечая, что диагональные матрицы $Q(t)-\varepsilon \mathfrak{A}_{0}(t)$ и $W_{0}(t, \varepsilon)$ коммутируют, получаем задачу Коши

$$
\dot{C}=-W_{0}^{-1}(t, \varepsilon) \mathfrak{A}_{*}(t) W_{0}(t, \varepsilon) C(t, \varepsilon), \quad C(0, \varepsilon)=I .
$$

Представим решение задачи (5.13) в виде

$$
C(t, \varepsilon)=I-\int_{0}^{t} W_{0}^{-1}(\tau, \varepsilon) \mathfrak{A}_{*}(\tau) W_{0}(\tau, \varepsilon) C(\tau, \varepsilon) d \tau .
$$

Подставив (5.14) в (5.11), находим, что оператор Коши $W(t, \varepsilon)$ уравнения (5.10) является решением интегрального уравнения

$$
W(t, \varepsilon)=W_{0}(t, \varepsilon)-\int_{0}^{t} W_{0}(t-\tau, \varepsilon) \mathfrak{A}_{*}(\tau) W(\tau, \varepsilon) d \tau
$$

ОПРЕДЕЛЕНИЕ 5.2. Матрица $Q(t)$ называется устойчивой на отрезке $[0, T]$, если при всех $0 \leqslant t \leqslant T$ выполняется неравенство

$$
\max _{1 \leqslant i \leqslant m} \operatorname{Re} \lambda_{i}(t) \leqslant-\alpha<0 .
$$


ПРЕДПОЛОЖЕНИЕ 5.2. Матрица $Q(t)$ является устойчивой на $[0, T]$.

Далее устанавливается, что при вьполненных предположениях 5.1, 5.2 уравнение (5.15) имеет единственное ограниченное решение $W(t, \varepsilon)$ при всех $0 \leqslant t \leqslant T$, причем

$$
\|W(t, \varepsilon)\| \leqslant N_{0} e^{-\left(\alpha / \varepsilon_{0}\right) t}+k \varepsilon_{0}, \quad 0 \leqslant t \leqslant T,
$$

где положительные постоянные $N_{0}, k$ не зависят от $\varepsilon$.

Таким образом, справедлива

ТЕОРема 5.1. Пусть для матрицы $Q(t)$ выполнены предположения 5.1, 5.2. Тогда оператор Коши $W(t, \varepsilon)$ уравнения (5.10) существует на всем отрезке $[0, T]$, удовлетворяет интегральному уравнению (5.15) и подчиняется оценке (5.17).

ЗАмЕчаниЕ 5.1. Предположение 5.1 в теореме 5.1 является основным. В то же время, не ограничивая общности, можно всегда считать вьполненным предположение 5.2.

5.3. Дифференциальное уравнение с трансформатором. Пусть для матрицы $Q(t)$ выполнены предположения 5.1, 5.2. Рассмотрим уравнение Риккати

$$
\varepsilon \dot{W}=(Q(t)-\varepsilon \mathfrak{A}(t)) W-W(Q(t)-\varepsilon F(t, \varepsilon))=\left(K_{0}(t)-\varepsilon T_{\mathfrak{A}(t), F(t, \varepsilon)}\right) W .
$$

Как и в $[21 ;$ гл. III, $\S 1$, п. 6], можно показать, что оператором Кошши для него служит трансформатор

$$
\mathscr{U}(t, \varepsilon) W=\mathscr{U}_{\mathrm{I}}(t, \varepsilon) W \mathscr{U}_{\mathrm{II}}(t, \varepsilon), \quad \mathscr{U}(0, \varepsilon) W=W
$$

где $\mathscr{U}_{\mathrm{I}}(t, \varepsilon)$ - оператор Коши уравнения (5.10), о котором шла речь в теореме 5.1 , а $\mathscr{U}_{\mathrm{II}}(t, \varepsilon)$ - оператор Коши вида (5.9) уравнения (5.8).

В самом деле, продифференцируем (5.19) по $t$ и умножим на $\varepsilon$, получим

$$
\begin{aligned}
\varepsilon \frac{d \mathscr{U}}{d t} W & =\varepsilon \frac{d \mathscr{U}_{\mathrm{I}}}{d t} W \mathscr{U}_{\mathrm{II}}+\mathscr{U}_{\mathrm{I}} W \varepsilon \frac{d \mathscr{U}_{\mathrm{II}}}{d t} \\
& =(Q-\varepsilon \mathfrak{A}) \mathscr{U}_{\mathrm{I}}(t, \varepsilon) W \mathscr{U}_{\mathrm{II}}(t, \varepsilon)-\mathscr{U}_{\mathrm{I}}(t, \varepsilon) W \mathscr{U}_{\mathrm{II}}(t, \varepsilon)(Q-\varepsilon F) \\
& =(Q-\varepsilon \mathfrak{A}) \mathscr{U}(t, \varepsilon) W-\mathscr{U}(t, \varepsilon) W(Q-\varepsilon F) .
\end{aligned}
$$

Отсюда следует, что любое решение неоднородного уравнения

$$
\varepsilon \dot{W}=\left(K_{0}(t)-\varepsilon T_{\mathfrak{A}(t), F(t, \varepsilon)}\right) W+\varepsilon \mathscr{R}(t, \varepsilon)
$$

можно записать в виде

$$
\begin{aligned}
W(t, \varepsilon) & =\mathscr{U}(t, \varepsilon) W(0, \varepsilon)+\int_{0}^{t} \mathscr{U}(t-s, \varepsilon) \mathscr{R}(s, \varepsilon) d s \\
& =\mathscr{U}_{\mathrm{I}}(t, \varepsilon) W(0, \varepsilon) \mathscr{U}_{\mathrm{II}}(t, \varepsilon)+\int_{0}^{t} \mathscr{U}_{\mathrm{I}}(t-s, \varepsilon) \mathscr{R}(s, \varepsilon) \mathscr{U}_{\mathrm{II}}(t-s, \varepsilon) d s .
\end{aligned}
$$




\section{§6. Матрица подобия $I+\xi Z(t, \xi)$}

Пусть матрица $Q(t)=\operatorname{diag}\left(q_{1}(t), \ldots, q_{m}(t)\right)$, имеющая простой спектр на отрезке $[0, T]$, возмушена некоторым мальм слагаемым, являющимся полной матрицей. В соответствии с п. 4.2 возьмем матрицу $Z(t, \xi)=Z_{*}(t, \xi)$ с нулевой диагональю и рассмотрим преобразование подобия $I+\xi Z(t, \xi)$, в котором $\xi$-мальй параметр.

6.1. Преобразование линейного пучка. В $\S 7$ речь пойдет о диагонализации с помощью матрицы $I+\xi Z(t, \xi)$ линейного матричного пучка $Q(t)-\xi \mathfrak{A}(t)$ при $q_{j}(t)=\lambda_{j}(t), 1 \leqslant j \leqslant m, \xi=\varepsilon$. Для корректности этой процедуры потребуем, чтобы при всех $0 \leqslant t \leqslant T$ выполнялось равенство

$$
(I+\xi Z)^{-1}\left((Q-\xi \mathfrak{A})(I+\xi Z)-\xi^{2} \dot{Z}\right)=Q-\xi^{\mathscr{G}},
$$

где $Q(t)-\xi \mathscr{G}(t, \xi)$ - некоторая диагональная матрица. Равенство (6.1) вьполнено, если сушествует единственное ограниченное решение дифференциального уравнения

$$
\xi \dot{Z}=\left(K_{0}(t)-\xi T_{\mathfrak{A}(t), \mathscr{G}(t, \xi)}\right) Z-\mathfrak{A}(t)+\mathscr{G}(t, \xi)
$$

относительно $Z(t, \xi)=Z_{*}(t, \xi)$ при всех $0 \leqslant t \leqslant T$ и всех достаточно малых $\xi$.

ЗАмечАние 6.1. Если матрица $\mathfrak{A}(t)$ оказывается диагональной в базисе $\left\{e_{r}\right\}$, то считаем $Z_{*}(t, \xi) \equiv 0$.

Примем $\xi=0$ в (6.2), получим матричное уравнение

$$
K_{0}(t) Z_{*}(t, 0)=\mathfrak{A}(t)-\mathscr{G}(t, 0),
$$

из которого согласно лемме 4.3 находим

$$
Q_{1}(t)=\mathscr{G}(t, 0)=\mathfrak{A}_{0}(t), \quad Z_{*}^{(1)}(t)=Z_{*}(t, 0)=\widehat{K}_{0}^{-1}(t) \mathfrak{A}_{*}(t) .
$$

Следовательно, в неизвестной пока матрице $\mathscr{G}(t, \xi)$ можно взять $Q_{1}(t)=\mathfrak{A}_{0}(t)=$ $\operatorname{diag}\left(\sigma_{1,1}(t), \ldots, \sigma_{m, m}(t)\right)$. Положим

$$
\mathscr{G}(t, \xi)=\mathfrak{A}_{0}(t)+\mathscr{G}_{0}(t, \xi), \quad Z_{*}(t, \xi)=Z_{*}^{(1)}(t)+\Psi(t, \xi),
$$

заметим, что $\mathscr{G}_{0}(t, 0) \equiv 0, \Psi(t, 0) \equiv 0$, и подставим (6.4) в (6.1). После преобразований от (6.1) перейдем к уравнению

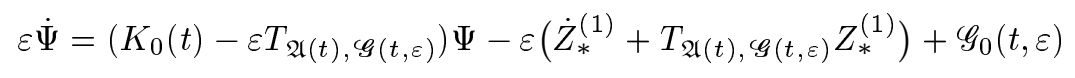

с $\mathscr{G}_{0}(t, 0) \equiv 0$. Если выполнено дополнительное предположение об устойчивости матрицы $Q(t)$ на $[0, T]$, то оператор Коши соответствующего однородного уравнения построен в п. 5.3. Тогда решение уравнения (6.5) сушествует на $[0, T]$, единственно и представимо по формуле (5.21). Значит, на $[0, T]$ существует единственное ограниченное решение дифференциального уравнения (6.2). Имеет место

ТЕОРема 6.1. Пусть матрица $Q(t)$ удовлетворяет предположениям 5.1, 5.2. Тогда существует единственное ограниченное на $[0, T]$ решение уравнения (6.1) при некоторой диагональной матрище $\mathscr{G}(t, \xi)$. 
6.2. Преобразование полиномиального пучка. В $\S 16$ (см. п. 16.1) предстоит от уравнения

$$
\xi^{n-1} \dot{\Psi}=(Q(t)-\xi G(t, \xi)) \Psi=\left(Q(t)-\sum_{i=1}^{m+n-2} \xi^{i} G^{(i)}(t)\right) \Psi, \quad \xi=\varepsilon^{1 / n}
$$

с полными матрицами $G^{(i)}(t)$ перейти к уравнению $\xi^{n-1} \dot{W}=(Q(t)-\xi \mathscr{G}(t, \xi)) W$ с диагональным пучком. Отметим, что оператор Коши уравнения (6.6) строится по схеме п. 5.2, и потребуем, чтобы при всех $0 \leqslant t \leqslant T$ выполнялось равенство

$$
(I+\xi Z)^{-1}\left((Q-\xi G)(I+\xi Z)-\xi^{n} \dot{Z}\right)=Q-\xi \mathscr{G}
$$

где $\mathscr{G}(t, \xi)$ - некоторая диагональная матрица. В свою очередь, равенство (6.7) будет выполняться, если сушествует единственное ограниченное решение дифференциального уравнения

$$
\xi^{n-1} \dot{Z}=\left(K_{0}(t)-\xi T_{G(t, \xi), \mathscr{G}(t, \xi)}\right) Z-G(t, \xi)+\mathscr{G}(t, \xi)
$$

относительно $Z(t, \xi)=Z_{*}(t, \xi)$ при всех $0 \leqslant t \leqslant T$ и всех достаточно малых $\xi$.

Действуя далее, как в п. 6.1, получаем следующее утверждение.

ТЕОРема 6.2. Пусть матрица $Q(t)$ удовлетворяет предположсениям 5.1, 5.2. Тогда существует единственное ограниченное на $[0, T]$ решение уравнения (6.7) при некоторой диагональной матриче $\mathscr{G}(t, \xi)$.

\section{§ 7. Об операторе Коши уравнения с малым параметром при производной. Случай простого спектра}

7.1. Уравнение в конечномерном пространстве. Обратимся к задаче Коши

$$
\varepsilon \dot{W}=\left(D_{0}(t)-\varepsilon D_{1}(t)\right) W, \quad 0 \leqslant t \leqslant T, \quad W(0, \varepsilon)=I,
$$

с матрицей $D_{0}(t)$, имеющей простой спектр. Уравнение $(7.1)$ служит непосредственнњм обобшением уравнения (5.10).

Как в п. 2.1, введем обратимую матрицу $\Phi(t)$, а также матрицу $\mathfrak{H}(t)$. Спектры подобных матрищ $D_{0}(t)$ и $Q(t)$ совпадают и

$$
Q(t)=\Phi^{-1}(t) D_{0}(t) \Phi(t), \quad \mathfrak{H}(t)=\Phi^{-1}(t) \dot{\Phi}(t)
$$

Будем искать решение задачи (7.1) в форме

$$
W(t, \varepsilon)=\mathfrak{S}(t, \varepsilon) W_{0}(t, \varepsilon) \mathfrak{S}^{-1}(0, \varepsilon), \quad W_{0}(0, \varepsilon)=I,
$$

где $W_{0}(t, \varepsilon)$ - оператор Коши уравнения (5.3), а оператор-функция $\mathfrak{S}(t, \varepsilon)$ подлежит определению в дальнейшем. Подставив (7.3) в (7.1), приходим к равенству

$$
\varepsilon \dot{W}_{0}=\mathfrak{S}^{-1}(t, \varepsilon)\left(\left(D_{0}(t)-\varepsilon D_{1}(t)\right) \mathfrak{S}(t, \varepsilon)-\varepsilon \dot{\mathfrak{S}}(t, \varepsilon)\right) W_{0} .
$$


На первом шаге алгоритма полагаем $\mathfrak{S}(t, \varepsilon)=\Phi(t) \mathfrak{S}_{\mathrm{I}}(t, \varepsilon)$. Тогда

$$
\Phi^{-1}(t) \varepsilon \dot{\mathfrak{S}}(t, \varepsilon)=\varepsilon \mathfrak{H}(t) \mathfrak{S}_{\mathrm{I}}(t, \varepsilon)+\varepsilon \dot{\mathfrak{S}}_{\mathrm{I}}(t, \varepsilon),
$$

откуда в согласии с (2.4) получаем

$$
\varepsilon \dot{W}_{0}=\mathfrak{S}_{\mathrm{I}}^{-1}(t, \varepsilon)\left((Q(t)-\varepsilon \mathfrak{A}(t)) \mathfrak{S}_{\mathrm{I}}(t, \varepsilon)-\varepsilon \dot{\mathfrak{S}}_{\mathrm{I}}(t, \varepsilon)\right) W_{0}
$$

Матрица $D_{0}(t)$ является диагональной в базисе $\left\{\varphi_{r}\right\}$. Привлечение матрицы $\Phi(t)$ дало возможность диагонализовать матрицу $D_{0}(t)$ в базисе $\left\{e_{r}\right\}$, получив вместо нее $Q(t)$.

На втором шаге алгоритма примем $\mathfrak{S}_{\mathrm{I}}(t, \varepsilon)=I+\varepsilon Z_{*}(t, \varepsilon)$, где $Z_{*}(t, \varepsilon)-$ матрица с нулевой диагональю (см. п. 4.3). Поскольку $\varepsilon \dot{\mathfrak{S}}_{\mathrm{I}}(t, \varepsilon)=\varepsilon^{2} \dot{Z}_{*}(t, \varepsilon)$, то $(7.5)$ записьвается в форме

$$
\varepsilon \dot{W}_{0}=\left(I+\varepsilon Z_{*}\right)^{-1}\left((Q-\varepsilon \mathfrak{A})\left(I+\varepsilon Z_{*}\right)-\varepsilon^{2} \dot{Z}_{*}\right) W_{0} .
$$

Чтобы с помощью матрицы $I+\varepsilon Z_{*}(t, \varepsilon)$ диагонализовать в базисе $\left\{e_{r}\right\}$ младшие члены матричного пучка, потребуем выполнения равенства (6.1) с $\xi=\varepsilon, \mathscr{G} \equiv F$ при всех $0 \leqslant t \leqslant T$ и всех достаточно малых $\varepsilon, 0<\varepsilon \leqslant \varepsilon_{0}$. Тогда уравнение (7.6) примет вид рассмотренной вьше задачи (5.3). Это произойдет, если существует единственное ограниченное решение дифференциального уравнения (6.2). Таким образом, мы оказались в условиях п. 6.1, согласно которому справедлива

ТЕОРема 7.1. Пусть матрица $D_{0}(t)$ удовлетворяет предположсениям 5.1, 5.2. Тогда решением задачи (7.1) является матричный пучок (7.3), в котором

$$
\mathfrak{S}(t, \varepsilon)=\Phi(t)\left(I+\varepsilon Z_{*}(t, \varepsilon)\right),
$$

а матрица $W_{0}(t, \varepsilon)$ определяется из следствия 5.2 .

В частном случае, когда в (7.1) матрица $D_{0}(t)$ является диагональной в базиce $\left\{e_{r}\right\}$, т.е. ког да $D_{0}(t)=Q(t)$, вместо матрицы $\Phi(t)$ следует взять тождественное преобразование $I$ и положить $\mathfrak{A}(t)=D_{1}(t)$. Тогда

$$
W(t, \varepsilon)=\left(I+\varepsilon Z_{*}(t, \varepsilon)\right) W_{0}(t, \varepsilon)\left(I+\varepsilon Z_{*}(0, \varepsilon)\right)^{-1}
$$

СЛЕДСТВИЕ 7.1. Если матрица $D_{0}(t)$ имеет простой спектр и $к$ тому же является диагональной, то $\mathfrak{A}(t)=D_{1}(t)$ и решение задачи (7.1) имеет вид (7.8).

7.2. Асимптотика оператора Коши. Пусть для матрицы $D_{0}(t)$ выполнено предположение 5.1. Получим явный вид решения уравнения

$$
\varepsilon \dot{Z}=\left(K_{0}(t)-\varepsilon T_{\mathfrak{A}(t), F(t, \varepsilon)}\right) Z-\mathfrak{A}(t)+F(t, \varepsilon),
$$

положив

$$
Z_{*}(t, \varepsilon)=\sum_{i=0}^{\infty} \varepsilon^{i} Z_{*}^{(i+1)}(t) .
$$


Для нахождения неизвестных матриц $Z_{*}^{(i)}(t), Q_{i}(t), i \in \mathbb{N}$, запишем $(7.9)$ в виде

$$
\begin{aligned}
\varepsilon \dot{Z}_{*}= & \left(K_{0}-\varepsilon T_{\mathfrak{A}, Q_{1}}\right) Z_{*}+\varepsilon^{2} Z_{*}\left(Q_{2}+\varepsilon Q_{3}+\varepsilon^{2} \cdots\right) \\
& +\left(Q_{1}-\mathfrak{A}\right)+\varepsilon Q_{2}+\varepsilon^{2} Q_{3}+\varepsilon^{3} \cdots
\end{aligned}
$$

и приравняем коэффициенты при $\varepsilon^{i}, i \in \mathbb{N}$. В результате придем к системе уравнений

$$
\begin{aligned}
& K_{0} Z_{*}^{(1)}=\mathfrak{A}-Q_{1}, \\
& K_{0} Z_{*}^{(2)}=\dot{Z}_{*}^{(1)}+T_{\mathfrak{A}, Q_{1}} Z_{*}^{(1)}-Q_{2}, \\
& K_{0} Z_{*}^{(3)}=\dot{Z}_{*}^{(2)}+T_{\mathfrak{A}, Q_{1}} Z_{*}^{(2)}-Z_{*}^{(1)} Q_{2}-Q_{3},
\end{aligned}
$$

Будем решать их последовательно с помошью леммы 4.3. Поскольку $Q(t)=$ $\operatorname{diag}\left(\lambda_{1}(t), \ldots, \lambda_{m}(t)\right)$ и $\mathfrak{A}(t)=\left(\sigma_{i j}(t)\right)$, то, как указано в п. 4.2,

$$
\widehat{K}_{0}^{-1} \mathfrak{A}_{*}(t)=\left(\left(\lambda_{i}(t)-\lambda_{j}(t)\right)^{-1} \sigma_{i j}(t)\right), \quad i \neq j, \quad 1 \leqslant i \leqslant m, \quad 1 \leqslant j \leqslant m .
$$

В итерационной процедуре одновременно находятся матрицы $Q_{i}(t)$ из условий разрешимости уравнений (7.12), а также матрицы $Z_{*}^{(i)}(t)$, служашие решениями этих уравнений. Следовательно,

$$
\begin{aligned}
Q_{1} & =I_{0} \mathfrak{A}, & Z_{*}^{(1)} & =\widehat{K}_{0}^{-1} I_{*} \mathfrak{A}, \\
Q_{2} & =I_{0}\left(T_{\mathfrak{A}, Q_{1}} Z_{*}^{(1)}\right), & Z_{*}^{(2)} & =\widehat{K}_{0}^{-1} I_{*}\left(\dot{Z}_{*}^{(1)}+T_{\mathfrak{A}, Q_{1}} Z_{*}^{(1)}\right), \\
Q_{3} & =I_{0}\left(T_{\mathfrak{A}, Q_{1}} Z_{*}^{(2)}-Z_{*}^{(1)} Q_{2}\right), & Z_{*}^{(3)} & =\widehat{K}_{0}^{-1} I_{*}\left(\dot{Z}_{*}^{(2)}+T_{\mathfrak{A}, Q_{1}} Z_{*}^{(2)}-Z_{*}^{(1)} Q_{2}\right)
\end{aligned}
$$

и т.д. Таким образом, решение $W(t, \varepsilon)$ задачи $(7.1)$, заданное формулами (7.3), $(7.7)$, может быть определено с любой наперед заданной точностью. На основании леммы 5.1 получаем следуюшее утверждение.

ТЕОРема 7.2. Пусть матрица $D_{0}(t)$ удовлетворяет предположсению 5.1 . Тогда формальным решением задачи (7.1) является матричный пучок (7.3), в котором матрицы $Q_{i}, Z_{*}^{(i)}$ определяются соотношениями (7.13).

В прикладных задачах широкое применение находят формулы асимптотических разложений решений векторных ЛДУ, в которых используется асимптотика оператора Коши. Преобразуем $W(t, \varepsilon)$, получив вначале представление для $\left(I+\varepsilon Z_{*}(0, \varepsilon)\right)^{-1}$. С этой целью положим

$$
\left(I+\varepsilon Z_{*}(\varepsilon)\right)^{-1}=I+\varepsilon K_{1}+\varepsilon^{2} K_{2}+\varepsilon^{3} K_{3}+\varepsilon^{4} \cdots
$$

и найдем постоянные матрицы $K_{i}, i \in \mathbb{N}$, из равенства

$$
\left(I+\varepsilon Z_{*}^{(1)}+\varepsilon^{2} Z_{*}^{(2)}+\varepsilon^{3} \cdots\right)\left(I+\varepsilon K_{1}+\varepsilon^{2} K_{2}+\varepsilon^{3} K_{3}+\varepsilon^{4} \cdots\right)=I .
$$


Приравняем коэффициенты при $\varepsilon^{i}, i \in \mathbb{N}$, придем к системе

$$
\begin{aligned}
& K_{1}+Z_{*}^{(1)}=0 \\
& K_{2}+Z_{*}^{(1)} K_{1}+Z_{*}^{(2)}=0 \\
& K_{3}+Z_{*}^{(1)} K_{2}+Z_{*}^{(2)} K_{1}+Z_{*}^{(3)}=0
\end{aligned}
$$

Отсюда

$$
\begin{aligned}
& K_{1}=-Z_{*}^{(1)} \\
& K_{2}=-Z_{*}^{(2)}+\left(Z_{*}^{(1)}\right)^{2}, \\
& K_{3}=-Z_{*}^{(3)}+Z_{*}^{(2)} Z_{*}^{(1)}+Z_{*}^{(1)} Z_{*}^{(2)}-\left(Z_{*}^{(1)}\right)^{3},
\end{aligned}
$$

и, значит,

$$
\begin{aligned}
& \left(I+\varepsilon Z_{*}(0, \varepsilon)\right)^{-1}=I-\varepsilon Z_{*}^{(1)}(0)-\varepsilon^{2}\left(Z_{*}^{(2)}(0)-\left(Z_{*}^{(1)}(0)\right)^{2}\right) \\
& \quad-\varepsilon^{3}\left(Z_{*}^{(3)}(0)-Z_{*}^{(2)}(0) Z_{*}^{(1)}(0)-Z_{*}^{(1)}(0) Z_{*}^{(2)}(0)+\left(Z_{*}^{(1)}(0)\right)^{3}\right)-\varepsilon^{4} \cdots .
\end{aligned}
$$

Подставляя $(7.7),(7.14)$ в (7.3), получаем, что

$$
\begin{aligned}
W(t, \varepsilon)= & \Phi(t)\left(W_{0}-\varepsilon\left(W_{0} Z_{*}^{(1)}(0)-Z_{*}^{(1)}(t) W_{0}\right)-\varepsilon^{2}\left(W_{0} Z_{*}^{(2)}(0)-Z_{*}^{(2)}(t) W_{0}\right.\right. \\
& \left.\left.-\left(W_{0} Z_{*}^{(1)}(0)-Z_{*}^{(1)}(t) W_{0}\right) Z_{*}^{(1)}(0)\right)-\varepsilon^{3} \cdots\right) \Phi^{-1}(0) .
\end{aligned}
$$

Поскольку $W_{0}(0, \varepsilon)=I$ и $[I, A(t)]=0 \forall A(t) \in \mathfrak{N}$, то $W(0, \varepsilon)=I$.

Теорема 7.3. Если матрица $D_{0}(t)$ имеет простой спектр, то решение задачи (7.1) представимо в форме асимптотического разложсения (7.16) по цельм степеням $\varepsilon$ с матрицами $Q_{i}, Z_{*}^{(i)}$, определяемыми соотношения$\mathcal{M u}(7.13)$.

В частном случае, когда матрицы $D_{0}, D_{1}$ постоянные, матрицы $\Phi, \mathfrak{A}$ также являются постоянными и оператор Коши $W(t, \varepsilon)$ преврашается в матричную экспоненту $\exp \left(\varepsilon^{-1}\left(D_{0}-\varepsilon D_{1}\right) t\right)$.

СлЕДСТВИЕ 7.2. Если матрицы $D_{0}, D_{1}$ постоянные и $D_{0}$ имеет простой спектр, то формула (7.16) с учетом (5.6) принимает вид

$$
\begin{aligned}
& \exp \left(\varepsilon^{-1}\left(D_{0}-\varepsilon D_{1}\right) t\right)= \Phi \\
&\left(W_{0}-\varepsilon\left[W_{0}, Z_{*}^{(1)}\right]-\varepsilon^{2}\left(\left[W_{0}, Z_{*}^{(2)}\right]\right.\right. \\
&\left.\left.-\left[W_{0}, Z_{*}^{(1)}\right] Z_{*}^{(1)}\right)-\varepsilon^{3} \cdots\right) \Phi^{-1}
\end{aligned}
$$

ЗАмечание 7.1. Если матрища $I_{0} \mathfrak{A}(t)$ зависит от $t$, а разности $\lambda_{i}(t)-\lambda_{j}(t)$, $i \neq j$, вместе с матрицей $I_{*} \mathfrak{A}(t)$ не зависят от $t$, то $Z_{*}^{(1)}$ - постоянная матрица. При этом матрицы $Z_{*}^{(i)}(t), i=2,3, \ldots$, вообще говоря, не являются постоянньми. 
7.3. Уравнение в сепарабельном гильбертовом пространстве. Случай стабильного спектра. Пусть $E$ - сепарабельное гильбертово пространство, и пусть $A(t)$ - линейный замкнутый симметрический оператор, действующий при каждом $t \in[0, T]$ в $E$ и такой, что

1) область определения $D(A)$ оператора $A(t)$ не зависит от $t$ и плотна в $E$;

2 ) оператор $A(t)$ сильно непрерывен на $D(A)$ (см. [22; гл. II, $\S 1]$ );

3) задача Коши

$$
\varepsilon \dot{x}=A(t) x, \quad 0 \leqslant t \leqslant T, \quad x(0, \varepsilon)=x^{0} \in E,
$$

равномерно корректна $[22 ;$ гл. II, $\S 3]$ при любом $\varepsilon \in\left(0, \varepsilon_{0}\right]$;

4) спектр оператора $A(t)$ при всех $0 \leqslant t \leqslant T$ стабилен, т.е. удовлетворяет соотношениям

$$
\lambda_{i}(t) \neq \lambda_{j}(t), \quad i \neq j, \quad i, j \in \mathbb{N}
$$

5) при каждом $t \in[0, T]$ сушествует ограниченный оператор $A^{-1}(t)$.

Как известно [23; гл. IV, п. 53], $A(t)$ допускает матричное представление в некотором ортонормированном базисе, принадлежашем плотному в $E$ множеству $D(A)$. Рассмотрим задачу Коши

$$
\varepsilon \dot{u}=A(t) u+f(t), \quad 0 \leqslant t \leqslant T, \quad u(0, \varepsilon)=u^{0} \in E .
$$

Общее решение уравнения (7.19) будем, как обычно, искать в виде суммы общего решения $\bar{u}(t, \varepsilon)$ уравнения (7.18) и частного решения $u^{*}(t, \varepsilon)$ уравнения (7.19).

Непосредственно проверяется, что если потребовать от $A^{-1}(t)$ и функции $f(t)$ непрерывной дифференцируемости бесконечного порядка, то

$$
u^{*}(t, \varepsilon)=-\sum_{r=0}^{\infty} \varepsilon^{r}\left(A^{-1}(t) \frac{d}{d t}\right)^{r} A^{-1}(t) f(t) .
$$

Решение $\bar{u}(t, \varepsilon)$ ишем в форме

$$
\bar{u}(t, \varepsilon)=W(t, \varepsilon) c(\varepsilon)
$$

где $c(\varepsilon)$ - произвольный вектор, а оператор Коши $W(t, \varepsilon)$ является решением задачи

$$
\varepsilon \dot{W}=A(t) W, \quad 0 \leqslant t \leqslant T, \quad W(0, \varepsilon)=I .
$$

Схема построения оператора Коши $W(t, \varepsilon)$ и его асимптотики совпадает с соответствующими результатами из пп. 7.1, 7.2 с учетом очевидных обобщений и поправок. Так, оператор $W(t, \varepsilon)$ определен на $D(A)$ и ограничен при каждом фиксированном $t$. Следовательно, по непрерывности его можно расширить на все пространство $E$, сохранив прежнее обозначение [22; гл. II, §3]. Поскольку оператор $A(t)$ обратим при всех $0 \leqslant t \leqslant T$, то он преобразует любой ортонормированный базис пространства $E$ в другой базис (Рисса) из $E$. Выбирая собственные векторы $\varphi_{k}(t)$, отвечаюшие собственньм значениям $\lambda_{k}(t)$, в качестве базиса Рисса в $E$, построим из векторов $\varphi_{k}(t)$, записанных в столбцы, матрицу $\Phi(t)$, а также матрицы $\mathfrak{A}(t)=\Phi^{-1}(t) \dot{\Phi}(t), Q(t)=\Phi^{-1}(t) A(t) \Phi(t)$. В формуле $(7.3)$ для $W(t, \varepsilon)$ следует принять

$$
\begin{gathered}
W_{0}(t, \varepsilon)=\operatorname{diag}\left(e^{\mu_{1}(t, \varepsilon)}, e^{\mu_{2}(t, \varepsilon)}, \ldots, e^{\mu_{m}(t, \varepsilon)}, \ldots\right), \\
\mu_{i}(t, \varepsilon)=\varepsilon^{-1} \int_{0}^{t}\left(\lambda_{i}(\tau)-\sum_{r=1}^{\infty} \varepsilon^{r} q_{r, i}(\tau)\right) d \tau, \quad i \in \mathbb{N} .
\end{gathered}
$$


ТЕОРема 7.4. Формальным решением задачи (7.19) в пространстве $E$ c оператор-функцией $A(t)$, подчиняющейся условиям 1)-5), является оператор-функиия $W(t, \varepsilon)$ вида $(7.3)$, где $W_{0}(t, \varepsilon)$ определяется из $(7.22)$, а $\mu_{i}(t, \varepsilon)$ - из (7.23).

\section{§8. Матрица подобия $I+\Omega_{-n}(t)$}

Всюду в дальнейшем исследуется случай кратного спектра.

Обозначим через $U_{i}(t)$ однодиагональные матрицы (см. п. 3.2), дающие в сумме структурную матрицу $\mathfrak{A}(t)$ из $\mathfrak{N}$, и предположим, что $U_{1-m}(t) \equiv \cdots \equiv U_{-n}(t) \equiv$ 0 , т.е. матрица $U_{1-n}(t)$ является в определенном смысле главной. Преобразование подобия $I+\varepsilon \Omega_{-n}(t)$ позволяет (см. пример 4.1) выделить в $U_{1-n}(t)$ главную часть $\mathscr{D}_{1-n}(t)$ и избавиться от слагаемого $U_{1-n}^{(0)}(t) \neq 0$ с $\gamma_{1-n}^{(0)}(t) \equiv 0$ в представлении $U_{1-n}(t)=\mathscr{D}_{1-n}(t)+U_{1-n}^{(0)}(t)$, где $\mathscr{D}_{1-n}(t) \in M, U_{1-n}^{(0)}(t) \in \operatorname{Im} K_{1}(\mathrm{~cm}$. формулу (8.4)). С учетом логарифмического закона введем обозначения

$$
\begin{aligned}
U_{i, 0}(t)= & {\left[U_{i+n}(t), \Omega_{-n}(t)\right], \quad 1-m \leqslant i \leqslant m_{1}-1, \quad i \neq-n, } \\
& U_{-n, 0}(t)=\dot{\Omega}_{-n}(t)+\left[U_{0}(t), \Omega_{-n}(t)\right], \\
U_{i, 1}(t)= & \left(-\Omega_{-n}(t)\right) U_{i+2 n}(t) \Omega_{-n}(t), \quad 1-m \leqslant i \leqslant-s-1 .
\end{aligned}
$$

Напомним (см. (1.3)), что $s=n-m_{1}, n+m_{1}=m$, причем числа $s, m$ одинаковой четности. Всюду далее будем считать выполненным

ПрЕДПОЛОЖЕНИЕ 8.1. Справедливы неравенства $1 \leqslant s \leqslant m$, или $0 \leqslant m_{1} \leqslant$ $(m-1) / 2$, или $(m+1) / 2 \leqslant n \leqslant m$.

Из условия $s \geqslant 1$, записанного в форме $2(1-n) \leqslant 1-m$, вытекают тождества

$$
U_{1-2 n, 0}(t) \equiv U_{1-n}(t) \Omega_{-n}(t) \equiv 0, \quad \Omega_{-n}^{2}(t) \equiv 0 .
$$

Значит, при достаточно малых $\varepsilon$

$$
\left(I+\varepsilon \Omega_{-n}(t)\right)^{-1}=I-\varepsilon \Omega_{-n}(t)
$$

и, следовательно,

$$
\begin{aligned}
(I+ & \left.\varepsilon \Omega_{-n}(t)\right)^{-1}\left((J-\varepsilon \mathfrak{A}(t))\left(I+\varepsilon \Omega_{-n}(t)\right)-\varepsilon^{2} \dot{\Omega}_{-n}(t)\right) \\
& =J-\varepsilon\left(\mathscr{D}_{1-n}(t)+\sum_{i=2-n}^{m-1} U_{i}(t)\right)-\varepsilon^{2} \sum_{i=1-m}^{m_{1}-1} U_{i, 0}(t)-\varepsilon^{3} \sum_{i=1-m}^{-s-1} U_{i, 1}(t) .
\end{aligned}
$$

ЗАмечАнИЕ 8.1. Пусть $m_{1}=0$. Тогда $n=m, \Omega_{-m}(t) \equiv 0$, следовательно, $U_{1-m}^{(0)}(t) \equiv 0, \mathscr{D}_{1-m}(t) \equiv U_{1-m}(t)$, и преобразование $I+\varepsilon \Omega_{-m}(t)$ преврашается в тождественное. 


\section{§9. Случай общего положения}

Обозначим через $\sigma_{r, l}(t)$ элемент из $r$-й строки, $l$-го столбца структурной матрицы $\mathfrak{A}(t)$, а через $\gamma_{i}(t)$ (без указания $\mathfrak{A}$ в качестве верхнего индекса) сумму элементов однодиагональной матрицы $U_{i}(t)$, или ее “след", $1-m \leqslant i \leqslant m-1$.

Случай общего положения (СОП) охарактеризуем соотношениями

$$
\gamma_{1-m}(t) \equiv \cdots \equiv \gamma_{-n}(t) \equiv 0, \quad \gamma_{1-n}(t) \neq 0, \quad 0 \leqslant m_{1} \leqslant \frac{m-1}{2} .
$$

Напомним, что во введении случай I), когда $m_{1}=0, n=m$,

$$
\gamma_{1-m}(t)=\sigma_{m, 1}(t) \neq 0,
$$

назван невырожденным, случай II), когда $m_{1}=1, n=m-1, m \geqslant 3$,

$$
\sigma_{m, 1}(t) \equiv 0, \quad \gamma_{2-m}(t)=\sigma_{m-1,1}(t)+\sigma_{m, 2}(t) \neq 0,
$$

назван случаем слабого вырождения. При $2 \leqslant m_{1} \leqslant(m-1) / 2$ говорят о случаях сильного вырождения матрицы $\mathfrak{A}(t)$.

$\mathrm{B} \S 8$ было показано, что преобразование подобия $I+\varepsilon \Omega_{-n}(t)$ с матрицей $\Omega_{-n}(t)$ из (4.3) приводит матрицу $\mathfrak{A}(t)$, у которой однодиагональная матрица $U_{i}(t) \neq 0$ с наименьшим номером $i$ (наибольшим "весом") имеет нулевой "след" $\gamma_{i}(t) \equiv 0$, к матрице с $U_{i}(t) \equiv 0$. Вследствие такой возможности будем всюду в дальнейшем характеризовать СОП несколько более жесткими по сравнению с (9.1) ограничениями

$$
U_{1-m}(t) \equiv \cdots \equiv U_{-n}(t) \equiv 0, \quad \gamma_{1-n}(t) \neq 0, \quad 0 \leqslant m_{1} \leqslant \frac{m-1}{2} .
$$

ЗАмечАние 9.1. Для случаев I), II) соотношения (9.1) и (9.2) совпадают.

\section{$\S 10$. Матрица подобия $\Lambda(\varepsilon)$}

Введем матрицу срезания [24]

$$
\Lambda(\varepsilon)=\operatorname{diag}\left(1, \varepsilon^{\nu}, \varepsilon^{2 \nu}, \ldots, \varepsilon^{(m-1) \nu}\right), \quad \nu>0,
$$

даюшую возможность отщепить от матрицы $\mathfrak{A}(t)$ однодиагональную матрицу $\mathscr{D}_{1-n}(t)$ с наименьшим индексом и возмутить ею матрицу $J$ так, чтобы у новой предельной матрицы пучка имелись различные собственные значения. Такого рода процедура в различных ситуациях использовалась, например, в [5], [14]; в [20] она названа присоединением к $J$ матрицы наибольшего веса, или перестройкой.

Матрица $\Lambda(\varepsilon)$ при действии на однодиагональные матрицы $X_{i}(t)$ обладает замечательным свойством:

$$
\Lambda^{-1}(\varepsilon) X_{i}(t) \Lambda(\varepsilon)=\varepsilon^{i \nu} X_{i}(t), \quad 1-m \leqslant i \leqslant m-1,
$$

в частности, $\Lambda^{-1} J \Lambda=\varepsilon^{\nu} J$. Формула (10.2) дает возможность ввести иерархию среди однодиагональных матриц, присваивая различные "веса" в зависимости от их номеров. 
Наряду с $\Lambda(\varepsilon)$ далее используется матрица срезания

$$
\bar{\Lambda}_{1}(\varepsilon)=\operatorname{diag}\left(1, \varepsilon^{\bar{\nu}}, \varepsilon^{2 \bar{\nu}}, \ldots, \varepsilon^{\left(m_{1}-1\right) \bar{\nu}}\right), \quad \bar{\nu}>0
$$

действующая в подпространстве End $P E$, а также матрицы

$$
\Lambda_{1}(\varepsilon)=\operatorname{diag}\left(\bar{\Lambda}_{1}(\varepsilon) ; 0_{n}\right), \quad \Lambda_{1}^{-1}(\varepsilon)=\operatorname{diag}\left(\bar{\Lambda}_{1}^{-1}(\varepsilon) ; 0_{n}\right),
$$

действующие в пространстве $\mathfrak{N}$.

Положим $\nu=1 / n$. Тогда

$$
\begin{aligned}
\Lambda^{-1}(\varepsilon)\left(J-\varepsilon\left(\mathscr{D}_{1-n}(t)+\sum_{i=2-n}^{m-1} U_{i}(t)\right)\right. \\
\left.\quad-\varepsilon^{2} \sum_{i=1-m}^{m_{1}-1} U_{i, 0}(t)-\varepsilon^{3} \sum_{i=1-m}^{-s-1} U_{i, 1}(t)\right) \Lambda(\varepsilon) \\
=\varepsilon^{1 / n}\left(\Gamma(t)-\sum_{i=1}^{m+n-2} \varepsilon^{i / n} H_{i+1-n}(t)\right)
\end{aligned}
$$

где для краткости использованы обозначения

$$
\begin{gathered}
\Gamma(t)=J-\mathscr{D}_{1-n}(t) ; \quad H_{i+1-n}(t)=U_{i+1-n}(t), \quad 1 \leqslant i \leqslant s-1 ; \\
H_{i+1-n}(t)=U_{i+1-n}(t)+U_{i+1-2 n, 0}(t), \quad s \leqslant i \leqslant s+n-1 ; \quad(10.5) \\
H_{i+1-n}(t)=U_{i+1-n}(t)+U_{i+1-2 n, 0}(t)+U_{i+1-3 n, 1}(t), \quad s+n \leqslant i \leqslant m+n-2 .
\end{gathered}
$$

ЗАмечАниЕ 10.1. Матрищы $\Omega_{-n}(t)$ и $\Lambda(\varepsilon)$ не коммутируют.

\section{§ 11. Матрица подобия $\Phi^{(1)}(t)$}

11.1. Корни степени $n$ из 1 . Свойства решений уравнения $q^{n}+\gamma(t)=0$, $n \geqslant 2$. Известно, что если $n \geqslant 2$, то $\sqrt[n]{1}=\exp (2 \pi j \sqrt{-1} / n), 1 \leqslant j \leqslant n$, причем одним из первообразных корней из единицы является число $e=\exp (2 \pi \sqrt{-1} / n)=$ $\cos (2 \pi / n)+\sqrt{-1} \sin (2 \pi / n)$, а все остальные корни равны $e^{j}$ и различны при разных $j$.

Лемма 11.1. Разности $e^{j}-e^{k}$ при некотором $k$ и всех $j, 1 \leqslant j \leqslant n, j \neq k$, попарно различны и не обращаются в нуль.

ЛЕМма 11.2. При всех $n \geqslant 2$ справедливо равенство

$$
\sum_{j=1}^{n} e^{j}=0
$$


Лемма 11.3. Если $p$ - челое число, $1 \leqslant|p| \leqslant n-1$, то при всех $n \geqslant 2$ справедливы равенства

$$
\sum_{j=1}^{n}\left(e^{j}\right)^{p}=\sum_{j=1}^{n-1}\left(e^{j}\right)^{p}=0 .
$$

Далее предположим, что $\gamma(t) \neq 0$ при всех $t \in[0, T]$, и рассмотрим алгебраическое уравнение $q^{n}+\gamma(t)=0, n \geqslant 2$. Его корнями служат $n$ различных функций

$$
q_{j}(t)=\sqrt[n]{-\gamma(t)} e^{j}, \quad 1 \leqslant j \leqslant n
$$

Следствиями леммы 11.3 являются

Лемма 11.4. Справедливы равенства

$$
\sum_{j=1}^{n}\left(\frac{q_{k}(t)}{q_{r}(t)}\right)^{j} \equiv 0, \quad 1 \leqslant|k-r| \leqslant n-1 .
$$

Лемма 11.5. Справедливъ равенства

$$
\sum_{j=1}^{n}\left(q_{j}(t)\right)^{p} \equiv 0, \quad 1 \leqslant|p| \leqslant n-1 .
$$

ЛЕмма 11.6. Отношение $\dot{q}_{j}(t) / q_{j}(t)$ не зависит от $j u$

$$
\frac{\dot{q}_{j}(t)}{q_{j}(t)}=\frac{\dot{\gamma}(t)}{n \gamma(t)}, \quad 1 \leqslant j \leqslant n
$$

11.2. Матрица $\Gamma(t)$. Предельной матрицей перестроенного пучка (см. (10.4)) является $\Gamma(t)=J-\mathscr{D}_{1-n}(t)$. Заметим, что если $m_{1}=0$, то выполняется равенство $\operatorname{det} \Gamma(t)=(-1)^{m} \sigma_{m, 1}(t) \neq 0$. В то же время $\operatorname{det} \Gamma(t) \equiv 0$ при $1 \leqslant m_{1} \leqslant(m-1) / 2$.

Найдем все собственные значения $q_{1}(t)$ матрицы $\Gamma(t)$. Проверяется, что уравнение $\operatorname{det}\left(\Gamma(t)-q_{1}(t) I\right) \equiv 0$ эквивалентно тождеству

$$
q_{1}^{m_{1}}\left(q_{1}^{n}+\gamma_{1-n}(t)\right) \equiv 0, \quad m_{1}+n=m .
$$

Следовательно,

$$
\begin{array}{lrl}
q_{1, j}(t)=\sqrt[n]{-\gamma_{1-n}(t)} \exp \left(\frac{2 \pi j \sqrt{-1}}{n}\right), & 1 \leqslant j \leqslant n, \\
q_{1, j}(t) \equiv 0, & n+1 \leqslant j \leqslant m .
\end{array}
$$

Таким образом, при $m_{1}=0$ и при $m_{1}=1$ все собственные значения матрицы $\Gamma(t)$ попарно различны. При $2 \leqslant m_{1} \leqslant(m-1) / 2$ у матрицы $\Gamma(t)$ имеются $n$ попарно различных ненулевых собственных значений и одно нулевое $m_{1}$-кратное собственное значение. 
11.3. Матрица Вандермонда и ее обобщение. Введем в рассмотрение обратимую матрицу $\Phi^{(1)}(t)$, играющую роль преобразования подобия, с помощью которой удается перейти от матрицы $\Gamma(t)$ к подобной ей матрице $\Gamma^{(1)}(t)$, записанной в ортонормированном базисе $\left\{e_{r}\right\}, 1 \leqslant r \leqslant m$. Предположим, что $q_{j}^{n}(t)=-\gamma_{1-n}(t) \neq 0$ при всех $0 \leqslant t \leqslant T, 1 \leqslant j \leqslant n$, и введем в рассмотрение $n$-мерные вектор-строки и вектор-столбцы

$$
\chi_{i}(t)=\left(q_{1}^{i}(t), \ldots, q_{n}^{i}(t)\right), \quad \theta_{i}(t)=\operatorname{col}\left(q_{1}^{i}(t), \ldots, q_{n}^{i}(t)\right), \quad i \in \mathbb{Z}
$$

где $\mathbb{Z}$ - множество всех целых чисел. Составим матрицу Вандермонда $\mathscr{V}(t)$ порядка $n \times n, n \geqslant 2$, строками которой служат $\chi_{i}(t), 0 \leqslant i \leqslant n-1$. Проверяется, что столбцами обратной матрицы $\mathscr{V}^{-1}(t)$ являются векторы $(1 / n) \theta_{-i}(t), 0 \leqslant i \leqslant n-1$.

В дальнейшем будут использоваться матрицы блочной структуры. В пространстве $\mathfrak{N}$ введем проекторы

$$
P=\operatorname{diag}\left(I_{m_{1}} ; 0_{n}\right), \quad I-P=\operatorname{diag}\left(0_{m_{1}} ; I_{n}\right)
$$

и станем обозначать блоки любой матрицы $A(t), 0 \leqslant t \leqslant T$, следующим образом:

$$
A_{11}=P A P, \quad A_{12}=P A(I-P), \quad A_{21}=(I-P) A P, \quad A_{22}=(I-P) A(I-P) .
$$

Далее, рассмотрим матрицу $\Phi^{(1)}(t)$ порядка $m \times m, m \geqslant n$, столбцами которой служат орты $e_{1}, \ldots, e_{m_{1}}$, а также векторы $\omega_{1}(t), \ldots, \omega_{n}(t)$, где $\omega_{j}(t)=$ $\operatorname{col}\left(1, q_{j}(t), \ldots, q_{j}^{m-1}(t)\right), 1 \leqslant j \leqslant n$. Последние $n$ векторов образуют блок размеров $m \times n$, строками которого служат $\chi_{i}(t), 0 \leqslant i \leqslant m-1$. При $n=m, m_{1}=0$ получаем $\Phi^{(1)}(t)=\mathscr{V}(t)$, поэтому $\Phi^{(1)}(t)$ - обобшение матрицы Вандермонда. Разложением по элементам первых $m_{1}$ столбцов устанавливается, что

$$
\operatorname{det} \Phi^{(1)}(t)=\left(\prod_{j=1}^{n} q_{j}^{m_{1}}(t)\right) \operatorname{det} \mathscr{V}(t) \neq 0 .
$$

Поскольку $0 \leqslant m_{1} \leqslant(m-1) / 2$, то

$$
\left(\Phi^{(1)}(t)\right)^{-1}=\left(\begin{array}{ccc}
I_{m_{1}} & 0_{m_{1} \times s} & \left(\frac{1}{\gamma_{1-n}(t)}\right) I_{m_{1}} \\
0_{n \times m_{1}} & \left(\Phi^{(1)}(t)\right)_{22}^{-1}
\end{array}\right),
$$

где $\left(\Phi^{(1)}(t)\right)_{22}^{-1}=(1 / n)\left(\theta_{-m_{1}}(t), \ldots, \theta_{1-m}(t)\right)$ - матрица порядка $n \times n$ вида

$$
\left(\Phi^{(1)}(t)\right)_{22}^{-1}=\frac{1}{n}\left(\begin{array}{ccc}
q_{1,1}^{-m_{1}}(t) & \ldots & q_{1,1}^{1-m}(t) \\
q_{1,2}^{-m_{1}}(t) & \ldots & q_{1,2}^{1-m}(t) \\
\ldots \ldots \ldots \ldots & \ldots \ldots \ldots \ldots \\
q_{1, n}^{-m_{1}}(t) & \ldots & q_{1, n}^{1-m}(t)
\end{array}\right) .
$$


11.4. Матрица $\Gamma^{(1)}(t)$. Обозначим

$$
\begin{aligned}
\Gamma^{(1)} & =\left(\Phi^{(1)}\right)^{-1} \Gamma \Phi^{(1)}, \quad H^{(0)}=\left(\Phi^{(1)}\right)^{-1} H_{0} \Phi^{(1)}+\mathfrak{A}^{(1)}, \\
H^{(i+1-n)} & =\left(\Phi^{(1)}\right)^{-1} H_{i+1-n} \Phi^{(1)}, \quad i \neq n-1, \quad 1 \leqslant i \leqslant m+n-2,
\end{aligned}
$$

где все матрицы зависят от $t$. Тогда согласно $(10.4),(11.5)$ имеем

$$
\begin{gathered}
\left(\Phi^{(1)}(t)\right)^{-1}\left(\left(\Gamma(t)-\sum_{i=1}^{m+n-2} \varepsilon^{i / n} H_{i+1-n}(t)\right) \Phi^{(1)}(t)-\varepsilon^{(n-1) / n} \dot{\Phi}^{(1)}(t)\right) \\
=\Gamma^{(1)}(t)-\sum_{i=1}^{m+n-2} \varepsilon^{i / n} H^{(i+1-n)}(t) .
\end{gathered}
$$

Устанавливается, что если $2 \leqslant m_{1} \leqslant(m-1) / 2$, то $\Gamma^{(1)}(t)=\operatorname{diag}\left(J_{1} ; Q(t)\right)$, где $J_{1}$ - матрица порядка $m_{1} \times m_{1}$ типа $J$, а $Q(t)=\operatorname{diag}\left(q_{1,1}(t), \ldots, q_{1, n}(t)\right)$. При $m_{1}=1$ имеем $\Gamma^{(1)}(t)=\operatorname{diag}(0 ; Q(t)), Q(t)=\operatorname{diag}\left(q_{1,1}(t), \ldots, q_{1, m-1}(t)\right)$. Таким образом, при $1 \leqslant m_{1} \leqslant(m-1) / 2$ предельная матрица $\Gamma^{(1)}(t)$ оказывается блочно-диагональной в базисе $\left\{e_{r}\right\}, 1 \leqslant r \leqslant m$. При $m_{1}=0$ имеется всего один блок и $\Gamma^{(1)}(t)=Q(t)=\operatorname{diag}\left(q_{1,1}(t), \ldots, q_{1, m}(t)\right)$.

\section{§ 12. Формулы для элементов блочных матриц}

Чтобы иметь возможность выразить коэффициенты расшепленных ЛДУ через коэффициенты исходного ЛДУ, нам потребуются представления блочных матриц $H_{k l}^{(i+1-n)}(t),-m_{1} \leqslant i \leqslant m+n-2, k, l=1,2$. В п. 12.1 рассмотрен случай $m_{1}=0$, в п. 12.2 - случай $m_{1}=1$, в п. 12.3 - случай $2 \leqslant m_{1} \leqslant(m-1) / 2$.

Обозначим через $X_{i+1-n}(t)$ матрицы, определяемње соотношениями

$$
\begin{aligned}
& X_{i+1-n}(t)=\sum_{r=1}^{m_{1}+1+i} x_{n-1+r-i, r}(t) V_{i+1-n}^{r-1, m_{1}+1+i-r}, \quad-m_{1} \leqslant i \leqslant n-1, \quad(12.1) \\
& X_{i+1-n}(t)=\sum_{r=1}^{m-1+n-i} x_{r, i+1+r-n}(t) V_{i+1-n}^{r-1, m+n-1-i-r}, \quad n-1 \leqslant i \leqslant m+n-2,
\end{aligned}
$$

а через $X^{(i+1-n)}(t)$ - подобные им матрицы $\left(\Phi^{(1)}\right)^{-1}(t) X_{i+1-n}(t) \Phi^{(1)}(t),-m_{1} \leqslant$ $i \leqslant m+n-2$, где в роли матрищы подобия будут выступать матрища Вандермонда $\mathcal{V}(t)$ или ее обобшение $\Phi^{(1)}(t)$ из п. 11.3. Приведем соответствующие формулы для элементов матриц $X_{k l}^{(i+1-n)}(t), k, l=1,2,-m_{1} \leqslant i \leqslant m+n-2$ (условимся опускать аргумент $t$ ).

12.1. Случай $m_{1}=0$. Заметим, что $n=m=s$, и обозначим через $\eta_{l, j}^{i+1-m}$ элемент матрицы $X^{(i+1-m)}=\mathscr{V}^{-1} X_{i+1-m} \mathscr{V}$, стоящий в $l$-й строке, $j$-м столбце, $l, j=1, \ldots, m, 0 \leqslant i \leqslant 2(m-1)$. 
Лемма 12.1. Справедливъ равенства

$$
\begin{array}{lrl}
\eta_{l, j}^{i+1-m}=\sum_{r=1}^{i+1} x_{r+m-1-i, r} \frac{\left(q_{j} / q_{l}\right)^{r-1}}{m q_{l}^{m-1-i}}, & 0 \leqslant i \leqslant m-1, \\
\eta_{l, j}^{i+1-m}=q_{j}^{i+1-m} \sum_{r=1}^{2 m-1-i} x_{r, r+i+1-m} \frac{\left(q_{j} / q_{l}\right)^{r-1}}{m}, & m-1 \leqslant i \leqslant 2(m-1) .
\end{array}
$$

СлЕДСТВИЕ 12.1. При $l=j$ имеем

$$
\begin{array}{lc}
\eta_{j, j}^{i+1-m}=\frac{\gamma_{i+1-m}^{X}}{m q_{j}^{m-1-i}}, & 0 \leqslant i \leqslant m-1, \\
\eta_{j, j}^{i+1-m}=q_{j}^{i+1-m} \frac{\gamma_{i+1-m}^{X}}{m}, & m-1 \leqslant i \leqslant 2(m-1),
\end{array}
$$

в частности,

$$
\eta_{j, j}^{0}=\frac{\gamma_{0}^{X}}{m}=\frac{1}{m} \operatorname{Tr} X
$$

12.2. Случай $m_{1}=1$. Здесь $n=m-1, s=m-2, P=P_{1}$ и имеет место

Лемма 12.2. Справедливы соотношения

$$
\begin{aligned}
X_{11}^{(1-m)} & =\frac{1}{\gamma_{1-n}} x_{m, 1} V_{0,1}^{0,0}, \\
X_{11}^{(0)} & =x_{1,1} V_{0,1}^{0,0}, \\
X_{11}^{(i+2-m)} & \equiv 0, \quad 0 \leqslant i \leqslant 2 m-3, \quad i \neq m-2 .
\end{aligned}
$$

Через $V_{0,1}^{0,0}$ обозначена матрица порядка $1 \times 1$ с элементом, равным 1 , т.е. $X_{11}^{(0)}$ - скаляр, равный $x_{1,1}$.

ЛЕмма 12.3. Справедливы соотношения

$$
\begin{array}{rlr}
X_{12}^{(i+2-m)} & =\frac{1}{\gamma_{1-n}} x_{m, i+2} \chi_{i+1}, & \\
X_{12}^{(0)} & =\left(x_{1,1}-x_{m, m}\right) \chi_{0}, & \\
X_{12}^{(i+2-m)} & =x_{1, i+3-m} \chi_{i+2-m}, & m-1 \leqslant i \leqslant 2 m-3 .
\end{array}
$$

Лемма 12.4. Справедливы соотночения

$$
\begin{array}{rlrl}
X_{21}^{(i+2-m)} & =\frac{1}{m-1} x_{m-1-i, 1} \theta_{i+2-m}, & & -1 \leqslant i \leqslant m-3, \\
X_{21}^{(i+2-m)} & \equiv 0, & m-2 \leqslant i \leqslant 2 m-3 .
\end{array}
$$

Далее, через $\eta_{l, j}^{i+2-m}$ обозначим элемент матрицы $X_{22}^{(i+2-m)}$ из $l$-й строки, $j$-го столбца, $l, j=1, \ldots, m-1,-1 \leqslant i \leqslant m-3$. 
ЛЕмма 12.5. Справедливъц соотношения

$$
\begin{array}{rlrl}
\eta_{l, j}^{i+2-m} & =\sum_{r=1}^{i+2} x_{r+m-2-i, r} \frac{\left(q_{j} / q_{l}\right)^{r-1}}{n q_{l}^{m-2-i}}, & & -1 \leqslant i \leqslant m-3, \\
\eta_{l, j}^{i+2-m} & =q_{j}^{i+2-m} \sum_{r=2}^{2(m-1)-i} x_{r, r+i+2-m} \frac{\left(q_{j} / q_{l}\right)^{r-1}}{n}, & & \\
\eta_{l, j}^{m-1} & \equiv 0 .
\end{array}
$$

СлЕДСТвИЕ 12.2. Eсли $l=j, m o$

$$
\begin{aligned}
\eta_{j, j}^{i+2-m}=\frac{\gamma_{i+2-m}^{X}}{n q_{j}^{n-1-i}}, & -1 \leqslant i \leqslant m-3, \\
\eta_{j, j}^{i+2-m}=\frac{1}{n} q_{j}^{i+2-m}\left(\gamma_{i+2-m}^{X}-x_{i+3-m, 1}\right), & m-2 \leqslant i \leqslant 2(m-2),
\end{aligned}
$$

в частности,

$$
\eta_{j, j}^{0}=\frac{1}{m-1} q_{j}^{i+2-m}\left(\operatorname{Tr} X-x_{1,1}\right)
$$

12.3. Случай $2 \leqslant m_{1} \leqslant(m-1) / 2$. Блок $X_{11}^{(i+1-n)}$ является однодиагональной (или, как ниже в (12.12), двухдиагональной) матрицей, поэтому он представляется с помощью базиса из $m_{1}^{2}$ матриц $V_{i-m_{1}, 1}^{r, i-1-r}, V_{m_{1}-i, 1}^{r, i-1-r}, 0 \leqslant r \leqslant i-1$, $1 \leqslant i \leqslant m_{1}$, порядка $m_{1} \times m_{1}$. При этом приходится различать следующие возможные соотношения между числами $s$ и $m_{1}$ :

a) $s \geqslant m_{1} \geqslant 2$, или $2 \leqslant m_{1} \leqslant m / 3$;

б) $s=m_{1}-1 \geqslant 1$, или $m_{1}=(m+1) / 3(m=3 l+2, l \in \mathbb{N})$;

в) $1 \leqslant s \leqslant m_{1}-2$, или $(m+2) / 3 \leqslant m_{1} \leqslant(m-1) / 2, m_{1} \geqslant 3$.

ЛЕмма 12.6. Если $s \geqslant m_{1} \geqslant 2$, то верны соотношения

$$
\begin{aligned}
X_{11}^{(i+1-n)} & =\frac{1}{\gamma_{1-n}} \sum_{r=1}^{m_{1}+1+i} x_{n-1+r-i, r} V_{i+1,1}^{r-1, m_{1}+1+i-r}, \quad-m_{1} \leqslant i \leqslant-1, \\
X_{11}^{(i+1-n)} & =\frac{1}{\gamma_{1-n}} \sum_{r=1}^{m_{1}-1-i} x_{r+n, r+i+1} V_{i+1,1}^{r-1, m_{1}-1-i-r}, \quad 0 \leqslant i \leqslant m_{1}-2, \\
X_{11}^{(i+1-n)} & \equiv 0, \quad m_{1}-1 \leqslant i \leqslant s-1, \\
X_{11}^{(i+1-n)} & =\sum_{r=1}^{i+1-s} x_{n-1+r-i, r} V_{i+1-n, 1}^{r-1, i+1-s-r}, \quad s \leqslant i \leqslant n-2, \\
X_{11}^{(i+1-n)} & =\sum_{r=1}^{m-1-i} x_{r, r+i+1-n} V_{i+1-n, 1}^{r-1, m-1-i-r}, \quad n-1 \leqslant i \leqslant m-2, \\
X_{11}^{(i+1-n)} & \equiv 0, \quad m-1 \leqslant i \leqslant m+n-2 .
\end{aligned}
$$


Лемма 12.7. Если $1 \leqslant s \leqslant m_{1}-2$, то наряду с (12.5), (12.9), (12.10) справедливы соотношения

$$
\begin{aligned}
X_{11}^{(i+1-n)}= & \frac{1}{\gamma_{1-n}} \sum_{r=1}^{m_{1}-1-i} x_{r+n, r+i+1} V_{i+1,1}^{r-1, m_{1}-1-i-r}, \quad 0 \leqslant i \leqslant s-1, \\
X_{11}^{(i+1-n)}= & \frac{1}{\gamma_{1-n}} \sum_{r=1}^{m_{1}-1-i} x_{r+n, r+i+1} V_{i+1,1}^{r-1, m_{1}-1-i-r} \\
& +\sum_{r=1}^{i+1-s} x_{r+n-1-i, r} V_{i+1-n, 1}^{r-1, i+1-r-s}, \quad 1 \leqslant s \leqslant i \leqslant m_{1}-2, \\
X_{11}^{(i+1-n)}= & \sum_{r=1}^{i+1-s} x_{r+n-1-i, r} V_{i+1-n, 1}^{r-1, i+1-r-s}, \quad m_{1} \leqslant i \leqslant n-2 .
\end{aligned}
$$

Лемма 12.8. Если $s=m_{1}-1$, то матрицъ $X_{11}^{(i+1-n)}$ определяются равенствами (12.5), (12.6), (12.8)-(12.10).

Отметим, что структура остальных блоков не зависит от соотношений между $s$ и $m_{1}$, и обозначим, как в лемме 12.5 , через $\eta_{l, j}^{i+1-n}$ элемент матрицы $X_{22}^{(i+1-n)}$, стоящий в $l$-й строке, $j$-м столбце $l, j=1, \ldots, n$.

Лемма 12.9. Справедливы соотношения

$$
\begin{aligned}
\eta_{l, j}^{i+1-n} & =\sum_{r=1}^{m_{1}+1+i} x_{r+n-1-i, r} \frac{\left(q_{j} / q_{l}\right)^{r-1}}{n q_{l}^{n-1-i}}, \quad-m_{1} \leqslant i \leqslant s-1, \\
\eta_{l, j}^{i+1-n} & =\sum_{r=i+2-s}^{m_{1}+1+i} x_{r+n-1-i, r} \frac{\left(q_{j} / q_{l}\right)^{r-1}}{n q_{l}^{n-1-i}}, \quad s \leqslant i \leqslant n-1, \\
\eta_{l, j}^{i+1-n} & =q_{j}^{i+1-n} \sum_{r=m_{1}+1}^{m+n-1-i} x_{r, r+i+1-n} \frac{\left(q_{j} / q_{l}\right)^{r-1}}{n}, \quad n-1 \leqslant i \leqslant 2(n-1), \\
\eta_{l, j}^{i+1-n} & \equiv 0, \quad 2 n-1 \leqslant i \leqslant m+n-2 .
\end{aligned}
$$

СлЕДСТвИЕ 12.3. При $l=j$ имеем

$$
\begin{aligned}
\eta_{j, j}^{i+1-n} & =\frac{\gamma_{i+1-n}^{X}}{n q_{j}^{n-1-i}}, \quad-m_{1} \leqslant i \leqslant s-1, \\
\eta_{j, j}^{i+1-n} & =\frac{1}{n q_{j}^{n-1-i}}\left(\gamma_{i+1-n}^{X}-\sum_{r=1}^{i+1-s} x_{r+n-1-i, r}\right), \quad s \leqslant i \leqslant n-1, \\
\eta_{j, j}^{i+1-n} & =\frac{q_{j}^{i+1-n}}{n}\left(\gamma_{i+1-n}^{X}-\sum_{r=1}^{m_{1}} x_{r, r+i+1-n}\right), \quad n-1 \leqslant i \leqslant 2(n-1),
\end{aligned}
$$

в частности,

$$
\eta_{j, j}^{-n}=\frac{\gamma_{-n}^{X}}{n q_{j}^{n}}=-\frac{\gamma_{-n}^{X}}{n \gamma_{1-n}}, \quad \eta_{j, j}^{0}=\frac{1}{n}\left(\operatorname{Tr} X-\sum_{r=1}^{m_{1}} x_{r, r}\right) .
$$

Блок $X_{12}^{(i+1-n)}$ размеров $m_{1} \times n$ описывается с помощью вектор-строк $\chi_{i}$. Имеет место 
Лемма 12.10. При $-m_{1} \leqslant i \leqslant-2$ первие $-i-1$ строк блока $X_{12}^{(i+1-n)}$ нулевые, остальные $m_{1}+1+i$ строк равны соответственно $\left(1 / \gamma_{1-n}\right) x_{n-i, 1} \chi_{0}, \ldots$, $\left(1 / \gamma_{1-n}\right) x_{m, i+m_{1}+1} \chi_{i+m_{1}}$.

При $-1 \leqslant i \leqslant s-1$ все $m_{1}$ строк блока $X_{12}^{(i+1-n)}$ равны соответственно $\left(1 / \gamma_{1-n}\right) x_{n+1, i+2} \chi_{i+1}, \ldots,\left(1 / \gamma_{1-n}\right) x_{m, i+m_{1}+1} \chi_{i+m_{1}}$.

При $s \leqslant i \leqslant n-1$ первые $n-1-i$ строк блока $X_{12}^{(i+1-n)}$ равны $\left(1 / \gamma_{1-n}\right) \times$ $x_{n+1, i+2} \chi_{i+1}, \ldots,\left(1 / \gamma_{1-n}\right) x_{2 n-1-i, n} \chi_{n-1}$, остальные $i+1-s$ строк равнь соответственно $\left(x_{n-i, 1}-x_{2 n-i, n+1}\right) \chi_{0}, \ldots,\left(x_{m_{1}, i+1-s}-x_{m, i+m_{1}+1}\right) \chi_{i-s}$.

При $n-1 \leqslant i \leqslant m-2$ первые $m-1-i$ строк блока $X_{12}^{(i+1-n)}$ равнь $\left(x_{1, i+2-n}-x_{n+1, i+2}\right) \chi_{i+1-n}, \ldots,\left(x_{m-1-i, m_{1}}-x_{m+n-1-i, m}\right) \chi_{m_{1}-1}$, остальнье $i+1-n$ строк равны соответственно $x_{m-i, m_{1}+1} \chi_{m_{1}}, \ldots, x_{m_{1}, i+1-s} \chi_{i-s}$.

При $m-1 \leqslant i \leqslant 2 n-1$ все $m_{1}$ строк блока $X_{12}^{(i+1-n)}$ равны соответственно $x_{1, i+2-n} \chi_{i+1-n}, \ldots, x_{m_{1}, i+1-s} \chi_{i-s}$.

Наконец, при $2 n \leqslant i \leqslant m+n-2$ первъе $m+n-i-1$ строк блока $X_{12}^{(i+1-n)}$ равны соответственно $x_{1, i+2-n} \chi_{i+1-n}, \ldots, x_{m+n-1-i, m} \chi_{m-1}$, a остальнье $i+1-2$ сторок состоят из нулей.

Блок $X_{21}^{(i+1-n)}$ размеров $n \times m_{1}$ описывается с помощью вектор-столбцов $\theta_{i}$. Справедлива

Лемма 12.11. При $-m_{1} \leqslant i \leqslant-2$ первые $m_{1}+1+i$ столбиов блока $X_{21}^{(i+1-n)}$ равны соответственно $(1 / n) x_{n-i, 1} \theta_{i+1-n}, \ldots,(1 / n) x_{m, m_{1}+1+i} \theta_{1-m}$, остальние $-i-1$ столбиов нулевие.

При $-1 \leqslant i \leqslant s-1$ все $m_{1}$ столбиов блока $X_{21}^{(i+1-n)}$ равны соответственно $(1 / n) x_{n-i, 1} \theta_{i+1-n}, \ldots,(1 / n) x_{m-1-i, m_{1}} \theta_{i+2-m}$.

При $s \leqslant i \leqslant n-2$ первые $i+1-s$ столбцов блока $X_{21}^{(i+1-n)}$ нулевые, а остальные $n-1-i$ столбиов равны соответственно $(1 / n) x_{m_{1}+1, i+2-s} \theta_{-m_{1}}, \ldots$, $(1 / n) x_{m-1-i, m_{1}} \theta_{i+2-m}$.

Наконеи, при $n-1 \leqslant i \leqslant m+n-2$ блок $X_{21}^{(i+1-n)}$ является нулевой матрицей.

\section{§ 13. Блочная структура матрицы $\mathfrak{A}^{(1)}(t)$}

Обозначим через $\mathfrak{A}^{(1)}(t)$ матрицу $\left(\Phi^{(1)}(t)\right)^{-1} \dot{\Phi}^{(1)}(t)$ и изучим ее блочную структуру, взяв в качестве $\Phi^{(1)}$ поочередно матрицу Вандермонда $\mathscr{V}$ и ее обобщение (см. п. 11.3).

13.1. Случай $m_{1}=0, n=m$. Матрица $(\mathscr{V}(t))^{-1} \dot{\mathscr{V}}(t)$ состоит из одного блока размеров $m \times m$. Проверяется, что ее элемент из $l$-й строки, $j$-го столбца равен

$$
\frac{\dot{\gamma}_{1-m}(t)}{m^{2} \gamma_{1-m}(t)} \sum_{r=1}^{m}(r-1)\left(\frac{q_{j}(t)}{q_{l}(t)}\right)^{r-1}, \quad l, j=1, \ldots, m
$$

В частности, при $l=j$ имеем

$$
\frac{m-1}{2 m} \frac{\dot{\gamma}_{1-m}(t)}{\gamma_{1-m}(t)}
$$


13.2. Случай $1 \leqslant m_{1} \leqslant(m-1) / 2$. Выделим блоки $\mathfrak{A}^{(1)}(t)$ в соответствии с (11.4). Поскольку первые $m_{1}$ столбцов матрицы $\dot{\Phi}^{(1)}(t)$ состоят из нулей, то $\mathfrak{A}_{11}^{(1)}(t) \equiv \mathfrak{A}_{21}^{(1)}(t) \equiv 0$. Строки блока $\mathfrak{A}_{12}^{(1)}(t)$ размеров $m_{1} \times n$ имеют вид $\left(-\dot{\gamma}_{1-n}(t) / \gamma_{1-n}(t)\right) \chi_{0}(t), \ldots,\left(-\dot{\gamma}_{1-n}(t) / \gamma_{1-n}(t)\right) \chi_{m_{1}-1}(t)$ (векторы $\chi_{i}(t)$ определены в п. 8.3). Элемент, стоящий в $l$-й строке, $j$-м столбце блока $\mathfrak{A}_{22}^{(1)}(t)$, равен

$$
\frac{\dot{\gamma}_{1-n}(t)}{n^{2} \gamma_{1-n}(t)} \sum_{r=m_{1}+1}^{m}(r-1)\left(\frac{q_{j}(t)}{q_{l}(t)}\right)^{r-1}, \quad l, j=1, \ldots, n .
$$

При $l=j$ найдем диагональные элементы

$$
\frac{m+m_{1}-1}{2 n} \frac{\dot{\gamma}_{1-n}(t)}{\gamma_{1-n}(t)}
$$

и отметим, что они не зависят от $j, 1 \leqslant j \leqslant n$.

\section{§14. Матрица подобия $I+\xi Y(t, \xi)$}

В этом параграфе будем предполагать, что $1 \leqslant m_{1} \leqslant(m-1) / 2$. Обозначим $\partial л я$ краткости

$$
\xi=\varepsilon^{1 / n}, \quad \varepsilon=\xi^{n}, \quad \frac{m+1}{2} \leqslant n \leqslant m,
$$

и условимся сохранять за матричнозначньми функциями прежние обозначения при замене аргумента $\varepsilon$ на $\xi$ и обратно.

14.1. Расщепление уравнения (14.7). Правая часть равенства (11.5) в новых обозначениях принимает вид

$$
\Gamma^{(1)}(t)-\sum_{i=1}^{m+n-2} \xi^{i} H^{(i+1-n)}(t)
$$

Матрицы $H^{(i+1-n)}(t), 1 \leqslant i \leqslant m+n-2$, в (14.2) не являются диагональными, поэтому представим их согласно обозначениям (11.4) в виде блочных матриц и введем в рассмотрение преобразование подобия $I+\xi Y(t, \xi)$, которое приведет младшие члены пучка к блочно-диагональному виду. С этой целью в согласии с (11.3) введем блочно-диагональную матрицу

$$
\mathfrak{B}(t, \xi)=\operatorname{diag}(F(t, \xi) ; G(t, \xi))=\sum_{i=1}^{m+n-2} \xi^{i-1} \mathfrak{B}^{(i)}(t)
$$

где

$$
\mathfrak{B}^{(i)}(t)=\operatorname{diag}\left(F^{(i)}(t) ; G^{(i)}(t)\right), \quad 1 \leqslant i \leqslant m+n-2 .
$$

Потребуем, чтобы при всех $0 \leqslant t \leqslant T$ выполнялось равенство

$$
(I+\xi Y)^{-1}\left(\left(\Gamma^{(1)}(t)-\sum_{i=1}^{m+n-2} \xi^{i} H^{(i+1-n)}(t)\right)(I+\xi Y)-\xi^{n} \dot{Y}\right)=\Gamma^{(1)}(t)-\xi \mathfrak{B}(t, \xi),
$$


в котором

$$
\begin{array}{ll}
\Gamma^{(1)}-\xi \mathfrak{B}=\operatorname{diag}\left(J_{1}-\xi F ; Q-\xi G\right), & \xi=\varepsilon^{1 / n}, \quad 2 \leqslant m_{1} \leqslant \frac{m-1}{2}, \\
\Gamma^{(1)}-\xi \mathfrak{B}=\operatorname{diag}(-\xi f ; Q-\xi G), & \xi=\varepsilon^{1 /(m-1)}, \quad m_{1}=1,
\end{array}
$$

причем $\xi f(t, \xi)=\sum_{i=1}^{m+n-2} \xi^{i} f^{(i)}(t)$ - скалярная функция. Равенство (14.5) представляет собой дифференциальное уравнение

$$
\xi^{n-1} \dot{Y}=\left[\Gamma^{(1)}(t), Y\right]-\xi T_{H(t, \xi), \mathfrak{B}(t, \xi)} Y-H(t, \xi)+\mathfrak{B}(t, \xi)
$$

относительно матричнозначной функции

$$
Y(t, \xi)=\sum_{i=1}^{m+n-2} \xi^{i-1} Y^{(i)}(t)
$$

Найденный в $\S 12$ вид блоков матриц $H^{(i+1-n)}(t)$ обусловливает структуру сплетающих матриц $Y^{(i)}(t), 1 \leqslant i \leqslant m+n-2$. Положим $Y_{11}(t, \xi) \equiv Y_{22}(t, \xi) \equiv 0$ и перепишем (14.7) в блочном виде. Воспользуемся легко проверяемыми соотношениями

$$
\begin{aligned}
& {\left[\Gamma^{(1)}(t), Y\right]_{11} \equiv 0, \quad\left[\Gamma^{(1)}(t), Y\right]_{22} \equiv 0,} \\
& {\left[\Gamma^{(1)}(t), Y\right]_{12}=T_{J_{1}, Q} Y_{12}, \quad\left[\Gamma^{(1)}(t), Y\right]_{21}=T_{Q, J_{1}} Y_{21} \text {; }} \\
& \left(T_{H, \mathfrak{B}} Y\right)_{11}=H_{12} Y_{21}, \quad\left(T_{H, \mathfrak{B}} Y\right)_{21}=T_{H_{22}, F} Y_{21}, \\
& \left(T_{H, \mathfrak{B}} Y\right)_{22}=H_{21} Y_{12}, \quad\left(T_{H, \mathfrak{B}} Y\right)_{12}=T_{H_{11}, G} Y_{12} \text {. }
\end{aligned}
$$

Тогда дифференциальное уравнение (14.7) распадается на два алгебраических и два дифференциальных уравнения вида

$$
\begin{aligned}
& \xi^{n-1} \dot{Y}_{21}=T_{Q, J_{1}} Y_{21}-\xi T_{H_{22}, F} Y_{21}-H_{21}, \quad F=H_{11}+\xi H_{12} Y_{21} \\
& \xi^{n-1} \dot{Y}_{12}=T_{J_{1}, Q} Y_{12}-\xi T_{H_{11}, G} Y_{12}-H_{12}, \quad G=H_{22}+\xi H_{21} Y_{12} .
\end{aligned}
$$

Теорема 14.1. Пусть $1 \leqslant m_{1} \leqslant(m-1) / 2$ в (9.2). Тогда уравнение (14.7) имеет единственное ограниченное на отрезке $[0, T]$ решение $Y(t, \xi)$ при любой матриче $H(t, \xi)$ и при некоторой матриче $\mathfrak{B}(t, \xi)$.

14.2. Асимптотика решения уравнения (14.7). Соотношения (14.10), (14.11) позволяют найти одновременно внедиагональные блоки $Y_{12}, Y_{21}$, а также матрицы $F, G$, являющиеся диагональными блоками в (14.7). Получим явный вид решений уравнений (14.10), (14.11), выбрав $Y(t, \xi)$ из (14.8).

Вернемся к вопросу о структуре матриц $Y^{(i)}(t), 1 \leqslant i \leqslant m+n-2$. В качестве строк матрицы $Y_{12}^{(i)}(t)$ порядка $m_{1} \times n$ выберем $n$-мерные вектор-строки $b_{i, 1}(t) \chi_{i+1}(t), b_{i, 2}(t) \chi_{i+2}(t), \ldots, b_{i, m_{1}}(t) \chi_{i+m_{1}}(t)$, а в качестве столбцов матрицы $Y_{21}^{(i)}(t)$ порядка $n \times m_{1}$ примем $n$-мерные вектор-столбцы $a_{i, 1}(t) \theta_{i+1}(t), a_{i, 2}(t) \theta_{i}(t)$, $\ldots, a_{i, m_{1}}(t) \theta_{i+2-m_{1}}(t)$, введенные в п. 11.3 в предположении, что при всех $0 \leqslant$ $t \leqslant T, 1 \leqslant j \leqslant n q_{1, j}^{n} \equiv-\gamma_{1-n}(t) \neq 0$. Здесь $a_{i, r}(t), b_{i, r}(t), 1 \leqslant i \leqslant m+n-2$, $1 \leqslant r \leqslant m_{1},-$ некоторые скалярные функции, поиск которых проводился в [15]. 
14.2.1. Случай $2 \leqslant m_{1} \leqslant(m-1) / 2$. Для нахождения неизвестных матрищ $Y_{12}^{(i)}(t), Y_{21}^{(i)}(t), F^{(i)}(t), G^{(i)}(t)$ представим компоненты $F, G$ матрицы $\mathfrak{B}$ в виде (14.3), (14.4) и приравняем коэффициенты при $\xi^{i}, 1 \leqslant i \leqslant m+n-2$, в (14.10), (14.11). В результате придем к рекуррентным соотношениям

$$
\begin{aligned}
F^{(i)}=H_{11}^{(i+1-n)}+\sum_{l=1}^{i-1} H_{12}^{(i+1-n-l)} Y_{21}^{(l)}, \quad 1 \leqslant i \leqslant m+n-2, \\
T_{Q, J_{1}} Y_{21}^{(i)}=H_{21}^{(i+1-n)}+\sum_{l=1}^{i-1} T_{H_{22}^{(i+1-n-l)}, F^{(i-l)} Y_{21}^{(l)}, \quad 1 \leqslant i \leqslant n-1,}{ }^{i-1} T_{J_{1}} Y_{21}^{(i)}=\dot{Y}_{21}^{(i+1-n)}+H_{21}^{(i+1-n)}+\sum_{l=1}^{(i+1-n-l)}, F^{(i-l)} Y_{21}^{(l)}, \\
n \leqslant i \leqslant m+n-2 ; \\
G^{(i)}=H_{22}^{(i+1-n)}+\sum_{l=1}^{i-1} H_{21}^{(i+1-n-l)} Y_{12}^{(l)}, \quad 1 \leqslant i \leqslant m+n-2, \\
T_{J_{1}, Q} Y_{12}^{(i)}=H_{12}^{(i+1-n)}+\sum_{l=1}^{i-1} T_{H_{11}^{(i+1-n-l)}, G^{(i-l)}} Y_{12}^{(l)}, \quad 1 \leqslant i \leqslant n-1, \\
T_{J_{1}, Q} Y_{12}^{(i)}=\dot{Y}_{12}^{(i+1-n)}+H_{12}^{(i+1-n)}+\sum_{l=1}^{i-1} T_{H_{11}^{(i+1-n-l)}, G^{(i-l)}} Y_{12}^{(l)}, \\
n \leqslant i \leqslant m+n-2 .
\end{aligned}
$$

С помощью лемм 4.1, 4.2 (см. формулы (4.1), (4.2)) можно отыскать любое наперед заданное число коэффициентов разложений по степеням $\xi$ матричных функций $Y_{21}(t, \xi), Y_{12}(t, \xi), F(t, \xi), G(t, \xi)$.

14.2.2. Случай $m_{1}=1$. Если $m_{1}=1$, или $n=m-1$, то

$$
Y_{12}^{(i)}=b_{i, 1}\left(q_{1,1}^{i+1}, \ldots, q_{1, m-1}^{i+1}\right), \quad Y_{21}^{(i)}=a_{i, 1} \operatorname{col}\left(q_{1,1}^{i+1}, \ldots, q_{1, m-1}^{i+1}\right)
$$

Вместо соотношений (14.13), (14.15), (14.12) получим соответственно

$$
\begin{aligned}
& Y_{21}^{(i)}=Q^{-1}\left(H_{21}^{(i+2-m)}+\sum_{l=1}^{i-1} T_{H_{22}^{(i+2-m-l)}, F^{(i-l)}} Y_{21}^{(l)}\right), \quad 1 \leqslant i \leqslant m-2, \\
& Y_{21}^{(i)}=Q^{-1}\left(\dot{Y}_{21}^{(i+2-m)}+H_{21}^{(i+2-m)}+\sum_{l=1}^{i-1} T_{H_{22}^{(i+2-m-l)}, F^{(i-l)}} Y_{21}^{(l)}\right), \quad \\
& \quad m-1 \leqslant i \leqslant 2 m-3 ; \\
& Y_{12}^{(i)}=-\left(H_{12}^{(i+2-m)}+\sum_{l=1}^{i-1} T_{H_{11}^{(i+2-m-l)}, G^{(i-l)}} Y_{12}^{(l)}\right) Q^{-1}, \quad 1 \leqslant i \leqslant m-2,
\end{aligned}
$$




$$
\begin{gathered}
Y_{12}^{(i)}=-\left(\dot{Y}_{12}^{(i+1-n)}+H_{12}^{(i+1-n)}+\sum_{l=1}^{i-1} T_{H_{11}^{(i+1-n-l)}, G^{(i-l)}} Y_{12}^{(l)}\right) Q^{-1} \\
m-1 \leqslant i \leqslant 2 m-3 \\
f^{(i)}(t)=H_{11}^{(i+2-m)}(t)+\sum_{l=1}^{i-1} H_{12}^{(i+2-m-l)}(t) Y_{21}^{(l)}(t), \quad 1 \leqslant i \leqslant 2 m-3 .
\end{gathered}
$$

Согласно леммам 12.2, 12.3, а также (14.16) имеем

$$
\begin{aligned}
H_{11}^{(i)}(t) \neq 0 \quad \Longleftrightarrow \quad i \geqslant 3-m, \quad i=k(m-1), & k=0,1,2, \ldots, \\
H_{12}^{(r)}(t) Y_{21}^{(i)}(t) \neq 0 & \Longleftrightarrow \quad r+i+1=k(m-1), \quad k=0,1,2, \ldots
\end{aligned}
$$

Это означает, что при $m_{1}=1$ имеем скалярное уравнение

$$
\dot{\psi}=-\sum_{i=0}^{2 m-3} \varepsilon^{i} f^{(i+1)}(t) \psi
$$

В [15] показано, что $H_{11}^{(0)}=\left(1 / \gamma_{2-m}\right)\left(\dot{\sigma}_{m-1,1}+\sigma_{1,1} \sigma_{m, 2}+\sigma_{m-1,1} \sigma_{m, m}\right)$, откуда находится главный член $-f^{(1)}(t)$ асимптотики решения, где

$$
f^{(1)}=H_{11}^{(0)}+\sum_{l=1}^{m-3} H_{12}^{(-l)} Y_{21}^{(l)}=\frac{1}{\gamma_{2-m}}\left(\dot{\sigma}_{m-1,1}+\sum_{k=1}^{m-1} \sigma_{k, 1} \sigma_{m, k+1}\right)
$$

$\S 15$. Об операторе Коши уравнения с малым параметром при производной. Случай кратного спектра

15.1. Постановка задачи. Рассмотрим задачу Коши

$$
\varepsilon \dot{W}=\left(D_{0}(t)-\varepsilon D_{1}(t)\right) W, \quad 0 \leqslant t \leqslant T, \quad W(0, \varepsilon)=I,
$$

с предельной матрицей $D_{0}(t)$, которая при всех $0 \leqslant t \leqslant T$ подобна матрице $\lambda(t) I+J$, где $J$ - жорданова клетка $m \times m$, отвечающая нулевому собственному значению.

ОПРЕДЕЛЕНИЕ 15.1. Матрица $D_{0}(t)$ назьвается устойчивой на отрезке $[0, T]$, если при всех $0 \leqslant t \leqslant T$ выполняется неравенство

$$
\operatorname{Re} \lambda(t) \leqslant-\alpha<0
$$


ПРЕДПОЛОЖЕНИЕ 15.1. Матрица $D_{0}(t)$ является устойчивой на $[0, T]$.

Пусть выполнены соотношения (9.2). Будем искать решение задачи (15.1) в форме

$$
W(t, \varepsilon)=e^{(1 / \varepsilon) \int_{0}^{t} \lambda(s) d s} \mathfrak{M}(t, \varepsilon) \Psi(t, \varepsilon) \mathfrak{M}^{-1}(0, \varepsilon), \quad \Psi(0, \varepsilon)=I,
$$

где матрица $\mathfrak{M}(t, \varepsilon)$ подлежит определению в дальнейшем. Матрица $\Psi(t, \varepsilon)$ в итоге будет блочно-диагональной, причем ее блоки являются операторами Коши соответственно двух уравнений

1) с предельной матрищей $J_{1}$ порядка $m_{1} \times m_{1}$, если $2 \leqslant m_{1} \leqslant(m-1) / 2$ (здесь $J_{1}$ - жорданова клетка того же типа, что $J$ );

2) с диагональной предельной матрицей, имеющей простой спектр.

При $m_{1}=1$ первое уравнение преврашается в скалярное и регулярно зависит от малого параметра. При $m_{1}=0$ первый блок, а вместе с ним и первое уравнение отсутствуют.

Подставив (15.2) в (15.1), придем к равенству

$$
\varepsilon \dot{\Psi}=\mathfrak{M}^{-1}(t, \varepsilon)\left(\left(D_{0}(t)-\lambda(t) I-\varepsilon D_{1}(t)\right) \mathfrak{M}(t, \varepsilon)-\varepsilon \dot{\mathfrak{M}}(t, \varepsilon)\right) \Psi .
$$

15.2. Первый шаг алгоритма. Используем формулы $(2.4),(2.5)$ и положим

$$
\mathfrak{M}(t, \varepsilon)=\Phi(t) \mathfrak{M}_{\mathrm{I}}(t, \varepsilon)
$$

в (15.3). Тогда согласно равенству $\Phi^{-1}(t) \varepsilon \dot{\mathfrak{M}}(t, \varepsilon)=\varepsilon \mathfrak{H}(t) \mathfrak{M}_{\mathrm{I}}(t, \varepsilon)+\varepsilon \dot{\mathfrak{M}}_{\mathrm{I}}(t, \varepsilon)$ получаем

$$
\varepsilon \dot{\Psi}=\mathfrak{M}_{\mathrm{I}}^{-1}(t, \varepsilon)\left((J-\varepsilon \mathfrak{A}(t)) \mathfrak{M}_{\mathrm{I}}(t, \varepsilon)-\varepsilon \dot{\mathfrak{M}}_{\mathrm{I}}(t, \varepsilon)\right) \Psi .
$$

С точностью до малого слагаемого нами получено уравнение с пучком, подобным исходному, в базисе $\left\{e_{r}\right\}, 1 \leqslant r \leqslant m$, причем главной матрицей пучка является $J$.

ЗАмечание 15.1 . Если с самого начала $D_{0}(t) \equiv J$, то в $(15.2)$ следует взять $\mathfrak{M}(t, \varepsilon)=\mathfrak{M}_{\mathbf{I}}(t, \varepsilon)$ и $\lambda(t) \equiv 0$.

15.3. Второй шаг алгоритма. Положим в (15.5)

$$
\mathfrak{M}_{\mathrm{T}}(t, \varepsilon)=\left(I+\varepsilon \Omega_{-n}(t)\right) \mathfrak{M}_{\mathrm{II}}(t, \varepsilon)
$$

и согласно равенствам (8.1), (8.3), (8.4) от (15.5) перейдем к эквивалентному уравнению

$$
\begin{aligned}
\varepsilon \dot{\Psi}= & \mathfrak{M}_{\mathrm{II}}^{-1}(t, \varepsilon)\left(\left(J-\varepsilon\left(\mathscr{D}_{1-n}(t)+\sum_{i=2-n}^{m-1} U_{i}(t)\right)-\varepsilon^{2} \sum_{i=1-m}^{m_{1}-1} U_{i, 0}(t)\right.\right. \\
& \left.\left.-\varepsilon^{3} \sum_{i=1-m}^{-s-1} U_{i, 1}(t)\right) \mathfrak{M}_{\mathrm{II}}(t, \varepsilon)-\varepsilon \dot{\mathfrak{M}}_{\mathrm{II}}(t, \varepsilon)\right) \Psi .
\end{aligned}
$$

Тем самьм в структурной матрице выделена главная часть $\mathscr{D}_{1-n}(t)$. 
15.4. Третий шаг алгоритма. Матрища $\mathscr{D}_{1-n}(t)$ участвует в перестройке пучка путем создания новой предельной матрицы $\Gamma(t)$. Одновременно происходит смена базиса $\left\{e_{r}\right\}, 1 \leqslant r \leqslant m$, на базис, составленный из собственных и присоединенных векторов матрицы $\Gamma(t)$. Положим в (15.6)

$$
\mathfrak{M}_{\mathrm{II}}(t, \varepsilon)=\Lambda(\varepsilon) \mathfrak{M}_{\mathrm{III}}(t, \varepsilon),
$$

воспользуемся равенствами (10.2), (10.4), (10.5). Тогда уравнение (15.6) записывается в форме

$$
\varepsilon^{(n-1) / n} \dot{\Psi}=\mathfrak{M}_{\mathrm{III}}^{-1}\left(\left(\Gamma(t)-\sum_{i=1}^{m+n-2} \varepsilon^{i / n} H_{i+1-n}(t)\right) \mathfrak{M}_{\mathrm{III}}-\varepsilon^{(n-1) / n} \dot{\mathfrak{M}}_{\mathrm{III}}\right) \Psi
$$

ЗАмЕчАниЕ 15.2 . Поскольку матрицы $\Omega_{-n}(t)$ и $\Lambda(\varepsilon)$ не коммутируют, то 2-й и 3 -й шаги алгоритма нельзя менять местами.

Равенство (15.7) в обозначениях (14.1) принимает вид

$$
\xi^{n-1} \dot{\Psi}=\mathfrak{M}_{\mathrm{III}}^{-1}\left(\left(\Gamma(t)-\sum_{i=1}^{m+n-2} \xi^{i} H_{i+1-n}(t)\right) \mathfrak{M}_{\mathrm{III}}-\xi^{n-1} \dot{\mathfrak{M}}_{\mathrm{III}}\right) \Psi .
$$

15.5. Четвертый шаг алгоритма. Вернемся в базис $\left\{e_{r}\right\}, 1 \leqslant r \leqslant m$, положив в $\left(15.7^{\prime}\right)$

$$
\mathfrak{M}_{\mathrm{III}}(t, \varepsilon)=\Phi^{(1)}(t) \mathfrak{M}_{\mathrm{IV}}(t, \varepsilon) .
$$

По аналогии с обозначением $(2.1)$ примем $\mathfrak{H}^{(1)}(t)=\left(\Phi^{(1)}(t)\right)^{-1} \dot{\Phi}^{(1)}(t)$ и заметим, что

$$
\left(\Phi^{(1)}(t)\right)^{-1} \xi^{n-1} \dot{\mathfrak{M}}_{\mathrm{III}}(t, \varepsilon)=\xi^{n-1} \mathfrak{H}^{(1)}(t) \mathfrak{M}_{\mathrm{IV}}(t, \varepsilon)+\xi^{n-1} \dot{\mathfrak{M}}_{\mathrm{IV}}(t, \varepsilon) .
$$

Кроме того, используем формулы (11.5). После преобразований уравнение $\left(15.7^{\prime}\right)$ записывается в форме

$$
\xi^{n-1} \dot{\Psi}=\mathfrak{M}_{\mathrm{IV}}^{-1}\left(\left(\Gamma^{(1)}(t)-\sum_{i=1}^{m+n-2} \xi^{i} H^{(i+1-n)}(t)\right) \mathfrak{M}_{\mathrm{IV}}-\xi^{n-1} \dot{\mathfrak{M}}_{\mathrm{IV}}\right) \Psi
$$

где, как указано в п. 11.4, $\Gamma^{(1)}(t)=\operatorname{diag}\left(J_{1} ; Q(t)\right)$ при $2 \leqslant m_{1} \leqslant(m-1) / 2$, $\Gamma^{(1)}(t)=\operatorname{diag}(0 ; Q(t))$ при $m_{1}=1$. Если $m_{1}=0, n=m$, то следует положить $\mathfrak{M}_{\mathrm{IV}}(t, \varepsilon)=I$, и тогда

$$
\varepsilon^{(m-1) / m} \dot{\Psi}=\left(Q(t)-\sum_{i=1}^{2(m-1)} \varepsilon^{i / m} H^{(i+1-m)}(t)\right) \Psi
$$

Уравнение $\left(15.8^{\prime}\right)$ соответствует случаю простого спектра при $D_{0}(t)=Q(t)$. Как и в $\S 7$, здесь следует диагонализовать младшие члены пучка, применив преобразование подобия $I+\xi Z_{*}(t, \xi)$. Позже в п. 16.2 мы вновь обратимся к случаю $m_{1}=0$. 
15.6. Пятый шаг алгоритма. Пусть $1 \leqslant m_{1} \leqslant(m-1) / 2$. Представим матрицы в форме блочно-диагональных, диагонализуем младшие члены пучка с помощью сплетающих матрищ $Y(t, \varepsilon)$, что позволит в конечном итоге расщепить уравнение. С этой целью примем $\mathfrak{M}_{V}(t, \varepsilon) \equiv I$ и положим

$$
\mathfrak{M}_{\mathrm{IV}}(t, \varepsilon)=I+\varepsilon^{1 / n} Y(t, \varepsilon)=I+\xi Y(t, \xi)
$$

в (15.8). Согласно равенству $\xi^{n-1} \dot{\mathfrak{M}}_{\mathrm{IV}}=\xi^{n} \dot{Y}$ вместо (15.8) получим эквивалентное уравнение

$$
\xi^{n-1} \dot{\Psi}=(I+\xi Y)^{-1}\left\{\left(\Gamma^{(1)}(t)-\sum_{i=1}^{m+n-2} \xi^{i} H^{(i+1-n)}(t)\right)(I+\xi Y)-\xi^{n} \dot{Y}\right\} \Psi
$$

Введем матрицу $\mathfrak{B}=\operatorname{diag}(F ; G)$, где $F(t, \xi), G(t, \xi)$ определены в (14.3), (14.4), и потребуем, чтобы выражение в фигурных скобках в (15.9) при всех $0 \leqslant t \leqslant T$ совпадало с матрицей $\Gamma^{(1)}-\xi \mathfrak{B}$ из (14.6). Далее представим $\Psi$ как блочно-диагональную матрицу $\operatorname{diag}\left(\Psi_{1} ; \Psi_{2}\right)$. Тогда уравнение $(15.9)$ при $2 \leqslant m_{1} \leqslant(m-1) / 2$ записывается в виде расщепленной системы

$\xi^{n-1} \dot{\Psi}_{1}=\left(J_{1}-\sum_{i=1}^{m+n-2} \xi^{i} F^{(i)}(t)\right) \Psi_{1}, \quad \xi^{n-1} \dot{\Psi}_{2}=\left(Q(t)-\sum_{i=1}^{m+n-2} \xi^{i} G^{(i)}(t)\right) \Psi_{2}$,

причем $\Psi_{1}(0, \xi)=I_{m_{1}}, \Psi_{2}(0, \xi)=I_{n}$.

ЗАмЕчАние 15.3. В результате всех пяти шагов алгоритма

$$
\mathfrak{M}(t, \varepsilon)=\Phi(t)\left(I+\varepsilon \Omega_{-n}(t)\right) \Lambda(\varepsilon) \Phi^{(1)}(t)\left(I+\varepsilon^{1 / n} Y(t, \varepsilon)\right)
$$

Если $m_{1}=1$, то первое уравнение в (15.10) с учетом (14.16) является скалярньм и принимает форму

$$
\dot{\psi}=-\sum_{i=0}^{2 m-3} \varepsilon^{i} f^{(i+1)}(t) \psi
$$

Обозначим $H(t, \xi)=\sum_{i=1}^{m+n-2} \xi^{i-1} H^{(i+1-n)}(t)$ и заметим, что переход от (15.9) к (15.10) возможен, если разрешимо дифференциальное уравнение (14.7) относительно сплетаюшей матрицы $Y(t, \xi)$ из (14.8). Записывая (14.7) в виде блоков и используя результаты $\S 14$, приходим к формулам (14.12)-(14.15).

Обратившись к системе (15.10), отметим, что первое уравнение напоминает с учетом замечания 15.1 исходное уравнение (15.1). Разница в том, что, во-первых, оно рассматривается не в $\mathfrak{N}$, а в подпространстве End $P E$ матриц размерности $m_{1} \times m_{1}$, зависяших от переменной $t, 0 \leqslant t \leqslant T$, во-вторых, изменился ранг малого параметра (показатель степени параметра при производной) и, в-третьих, правая часть разложена не по цельм степеням $\varepsilon$, а по степеням $\xi=\varepsilon^{1 / n}$. Что касается второго уравнения, то оно соответствует случаю простого спектра при $D_{0}(t)=Q(t)$ (см. также $\left(15.8^{\prime}\right)$, где $\left.n=m\right)$. 


\section{$\S 16$. Уравнение в подпространстве $\operatorname{End}(I-P) E$. Случай I $)$}

16.1. Представление для $W_{I-P}(t, \varepsilon)$. Действуя по схеме п. 6.2 , перейдем от уравнения

$$
\xi^{n-1} \dot{\Psi}_{2}=(Q(t)-\xi G(t, \xi)) \Psi_{2}=\left(Q(t)-\sum_{i=1}^{m+n-2} \xi^{i} G^{(i)}(t)\right) \Psi_{2}
$$

в подпространстве $\operatorname{End}(I-P) E$ к уравнению $\xi^{n-1} \dot{W}=(Q(t)-\xi \mathscr{G}(t, \xi)) W$ с диагональньм пучком в том же подпространстве. Такой переход возможен в силу теоремы 6.2 о существовании единственного ограниченного решения дифференциального уравнения (6.8) относительно матрицы $Z(t, \xi)=Z_{*}(t, \xi)$ с нулевой диагональю при некоторой диагональной матрице $\mathscr{G}(t, \xi)$ при всех $0 \leqslant t \leqslant T$ и всех достаточно малых $\xi$. В результате имеем

$$
W_{I-P}(t, \varepsilon)=\mathfrak{M}(t, \varepsilon) \operatorname{diag}\left(0_{m_{1}} ; e^{\widetilde{\mu}_{1}(t, \varepsilon)}, \ldots, e^{\widetilde{\mu}_{n}(t, \varepsilon)}\right) \mathfrak{M}^{-1}(0, \varepsilon),
$$

где

$$
\begin{aligned}
\mathfrak{M}(t, \varepsilon)= & \Phi(t)\left(I+\varepsilon \Omega_{-n}(t)\right) \Lambda(\varepsilon) \Phi^{(1)}(t)\left(I+\varepsilon^{1 / n} Y(t, \varepsilon)\right) \\
& \times\left(I+\varepsilon^{1 / n} Z_{*}(t, \varepsilon)\right) \\
\mathfrak{M}^{-1}(0, \varepsilon)= & O\left(\varepsilon^{(1-m) / n}\right) \\
\widetilde{\mu}_{j}(t, \varepsilon)= & \varepsilon^{-1} \int_{0}^{t}\left(\lambda(\tau)-\varepsilon^{1 / n} q_{1, j}(\tau)-\varepsilon^{2 / n} \frac{\gamma_{2-n}(\tau)}{n q_{1, j}^{n-2}(\tau)}-\varepsilon^{3 / n} \ldots\right) d \tau, \\
& 1 \leqslant j \leqslant n .
\end{aligned}
$$

16.2. Случай I). Так как $m_{1}=0$, или $n=m$, то, используя равенства $(2.5),(10.4),(11.5)$, после применения преобразования подобия $\Phi(t) \Lambda(\varepsilon) \mathscr{V}(t)$ получаем уравнение $\left(15.8^{\prime}\right)$, т.е. уравнение $(16.1)$ в $\operatorname{End} E$ при $\xi=\varepsilon^{1 / m}, G^{(i)}=$ $H^{(i+1-m)}, 1 \leqslant i \leqslant 2(m-1)$. Как и в $\S 7$, здесь следует диагонализовать младшие члены пучка, применив преобразование подобия $I+\xi Z_{*}(t, \xi)$. Напомним, что согласно (10.5) имеем

$$
\begin{gathered}
\Gamma(t)=J-\mathscr{D}_{1-m}(t) ; \quad H_{i+1-m}(t)=U_{i+1-m}(t), \quad 1 \leqslant i \leqslant m-1 ; \\
H_{i+1-m}(t)=U_{i+1-m}(t)+U_{i+1-2 m, 0}(t), \quad m \leqslant i \leqslant 2(m-1) .
\end{gathered}
$$

Из (11.4) находим

$$
\begin{gathered}
G^{(m-1)}(t)=H^{(0)}(t)=\mathscr{V}^{-1}(t)\left(U_{0}(t) \mathscr{V}(t)+\dot{\mathcal{V}}(t)\right), \\
H^{(i+1-m)}=\mathscr{V}^{-1}(t) H_{i+1-m}(t) \mathscr{V}(t), \\
i \neq m-1, \quad 1 \leqslant i \leqslant 2(m-1) .
\end{gathered}
$$


Матрицы $Z^{(i)}(t)$ имеют нулевую диагональ, а $Q_{i}(t)$ являются диагональньми. Для нахождения явного вида неизвестных матриц $Z^{(i)}(t), Q_{i}(t), i \in \mathbb{N}$, рассмотрим дифференциальное уравнение

$$
\begin{aligned}
& \left(Q-\sum_{i=1}^{2(m-1)} \xi^{i} H^{(i+1-m)}\right)\left(I+\sum_{i=1}^{\infty} \xi^{i} Z^{(i)}\right) \\
& =\left(I+\sum_{i=1}^{\infty} \xi^{i} Z^{(i)}\right)\left(Q-\sum_{i=1}^{2(m-1)} \xi^{i} Q_{i}\right)+\xi^{m} \sum_{i=1}^{\infty} \xi^{i} \dot{Z}^{(i)}
\end{aligned}
$$

относительно матриц $Z^{(i)}(t)$. Приравнивая коэффициенты при $\xi^{i}, i \in \mathbb{N}$, приходим к итерационной процедуре

$$
\begin{aligned}
& K_{0} Z^{(1)}=H^{(2-m)}-Q_{1} \\
& K_{0} Z^{(2)}=T_{H^{(2-m)}, Q_{1}} Z^{(1)}+H^{(3-m)}-Q_{2} \\
& K_{0} Z^{(3)}=T_{H^{(2-m)}, Q_{1}} Z^{(2)}+T_{H^{(3-m)}, Q_{2}} Z^{(1)}+H^{(4-m)}-Q_{3} \\
& \cdots \cdots \cdots \cdots \cdots \cdots \cdots \cdots \cdots \cdots \cdots \cdots \cdots \cdots \cdots \cdots \cdots \cdots \cdots \cdots \cdots \cdots \cdots \cdots \cdots \cdots \cdots \cdots \cdots \cdots \cdots \\
& K_{0} Z^{(m-1)}=\dot{Z}^{(1)}+T_{H^{(2-m)}, Q_{1}} Z^{(m-2)}+T_{H^{(3-m)}, Q_{2}} Z^{(m-3)}+\cdots \\
& \quad+T_{H^{(-1)}, Q_{m-2}} Z^{(1)}+H^{(0)}-Q_{m-1},
\end{aligned}
$$

Здесь одновременно находятся матрицы $Q_{i}(t)$ из условий разрешимости уравнений (16.3), а также матрицы $Z^{(i)}(t)$, служащие решениями этих уравнений. Так как $H^{(2-m)}=\left(h_{k l}^{(2-m)}\right)$, где

$$
h_{k l}^{(2-m)}=\frac{1}{m} q_{1, k}^{2-m}\left(\sigma_{m-1,1}+\frac{q_{1, l}}{q_{1, k}} \sigma_{m, 2}\right), \quad k, l=1,2, \ldots, m,
$$

то с учетом леммы 4.5 найдем $Q_{1}$ из условий разрешимости первого уравнения в (16.3):

$$
Q_{1}=\frac{\gamma_{2-m}}{m}\left(Q^{-1}\right)^{m-2} .
$$

В то же время по формуле (4.4) отышем элементы $z_{k l}^{(1)}$ матрицы $Z^{(1)}$ :

$$
z_{k l}^{(1)}=\frac{1}{m q_{1, k}^{m-2}\left(q_{1, k}-q_{1, l}\right)}\left(\sigma_{m-1,1}+\frac{q_{1, l}}{q_{1, k}} \sigma_{m, 2}\right), \quad k \neq l, \quad k, l=1,2, \ldots, m .
$$

Точно так же $Q_{2}$ находится из условий разрешимости второго уравнения в (16.3), и его решение $Z^{(2)}$ записывается согласно формуле (4.4). Продолжая аналогичные рассуж дения, можем найти любое наперед заданное число диагональных матриц $Q_{i}$.

Таким образом, при $m_{1}=0$ имеем

$$
\begin{aligned}
& \mathfrak{M}(t, \varepsilon)=\Phi(t) \Lambda(\varepsilon) \mathscr{V}(t)\left(I+\varepsilon^{1 / m} Z_{*}(t, \varepsilon)\right), \\
& W(t, \varepsilon)=\mathfrak{M}(t, \varepsilon) \operatorname{diag}\left(e^{\widetilde{\mu}_{1}(t, \varepsilon)}, \ldots, e^{\widetilde{\mu}_{m}(t, \varepsilon)}\right) \mathfrak{M}^{-1}(0, \varepsilon),
\end{aligned}
$$

где $\mathfrak{M}^{-1}(0, \varepsilon)=O\left(\varepsilon^{(1-m) / m}\right)$ и при всех $1 \leqslant j \leqslant m$

$$
\widetilde{\mu}_{j}(t, \varepsilon)=\varepsilon^{-1} \int_{0}^{t}\left(\lambda(\tau)-\varepsilon^{1 / m} q_{1, j}(\tau)-\varepsilon^{2 / m} \frac{\gamma_{2-m}(\tau)}{m q_{1, j}^{m-2}(\tau)}-\varepsilon^{3 / m} \ldots\right) d \tau
$$


ТЕОРема 16.1. Если въполнено предположение 15.1 и

$$
\sigma_{m, 1}(t) \neq 0, \quad m \geqslant 2
$$

при всех $0 \leqslant t \leqslant T$, то оператор Коши уравнения (15.1) имеет вид (16.10), причем матрица $\mathfrak{M}(t, \varepsilon)$ находится из (16.9) и скалярные функции $\widetilde{\mu}_{j}(t, \varepsilon)$ определяются равенствами (16.11).

В частности, когда $D(t, \varepsilon) \equiv D(\varepsilon)$, имеем

$$
\exp \left(\varepsilon^{-1} D(\varepsilon) t\right)=e^{(\lambda / \varepsilon) t} \mathfrak{M}(\varepsilon) \operatorname{diag}\left(e^{t \mu_{1}(\varepsilon)}, \ldots, e^{t \mu_{m}(\varepsilon)}\right) \mathfrak{M}^{-1}(\varepsilon)
$$

где

$$
\mu_{j}(\varepsilon)=-\varepsilon^{(1-m) / m}\left(q_{1, j}+\varepsilon^{1 / m} \frac{\gamma_{2-m}}{m q_{1, j}^{m-2}}+\varepsilon^{2 / m} \ldots\right), \quad 1 \leqslant j \leqslant m .
$$

\section{$\S 17$. Скалярное уравнение в End $P E$. Случай II)}

Пусть $m_{1}=1$ в (9.2). В подпункте 14.2 .2 указано, что после расшепления получаем одномерное (скалярное) уравнение (14.20), а также уравнение

$$
\xi^{m-2} \dot{\Psi}_{2}=\left(Q(t)-\sum_{i=1}^{2 m-3} \xi^{i} G^{(i)}(t)\right) \Psi_{2}
$$

в подпространстве $\operatorname{End}(I-P) E$ размерности $n=m-1$, в котором $\xi=\varepsilon^{1 /(m-1)}$, $G^{(i)}=H^{(i+2-m)}, 1 \leqslant i \leqslant 2 m-3$. После применения преобразования подобия $I+\xi Z(t, \xi)$ уравнение (17.1) примет вид

$$
\xi^{m-2} \dot{W}=\left(Q(t)-\sum_{i=1}^{2 m-3} \xi^{i} Q_{i}(t)\right) W
$$

Таким образом, при $m_{1}=1$ в итоге всех шести преобразований подобия находим $\mathfrak{M}(t, \varepsilon)$ из $(16.3)$, полагая $n=m-1$. Тогда

$$
W(t, \varepsilon)=\mathfrak{M}(t, \varepsilon) \operatorname{diag}\left(e^{\widetilde{\mu}_{m}(t, \varepsilon)} ; e^{\widetilde{\mu}_{1}(t, \varepsilon)}, \ldots, e^{\widetilde{\mu}_{m-1}(t, \varepsilon)}\right) \mathfrak{M}^{-1}(0, \varepsilon),
$$

где $\mathfrak{M}^{-1}(0, \varepsilon)=O\left(\varepsilon^{-1}\right)$,

$$
\begin{aligned}
\widetilde{\mu}_{j}(t, \varepsilon)= & \varepsilon^{-1} \int_{0}^{t}\left(\lambda(\tau)-\varepsilon^{1 /(m-1)} q_{1, j}(\tau)\right. \\
& \left.-\varepsilon^{2 /(m-1)} \frac{\gamma_{3-m}(\tau)}{(m-1) q_{1, j}^{m-3}(\tau)}-\varepsilon^{3 /(m-1)} \ldots\right) d \tau, \quad 1 \leqslant j \leqslant m-1 \\
\widetilde{\mu}_{m}(t, \varepsilon)= & \varepsilon^{-1} \int_{0}^{t}\left(\lambda(\tau)-\varepsilon f^{(1)}(\tau)-\varepsilon^{2} f^{(2)}(\tau)-\varepsilon^{3} \ldots\right) d \tau
\end{aligned}
$$

ТЕОРема 17.1. Если въполнено предположение 15.1 и

$$
\sigma_{m, 1}(t) \equiv 0, \quad \gamma_{2-m}(t) \neq 0, \quad m \geqslant 3
$$


при всех $0 \leqslant t \leqslant T$, то оператор Коши уравнения (15.1) имеет вид (17.3), причем матрича $\mathfrak{M}(t, \varepsilon)$ находится из (16.3) при $n=m-1$ и скалярные функции $\widetilde{\mu}_{j}(t, \varepsilon)$ определяются равенствами (17.4).

В частности, когда $D(t, \varepsilon) \equiv D(\varepsilon)$, получаем

$$
\exp \left(\varepsilon^{-1} D(\varepsilon) t\right)=e^{(\lambda / \varepsilon) t} \mathfrak{M} \operatorname{diag}\left(e^{t \mu_{m}(\varepsilon)} ; e^{t \mu_{1}(\varepsilon)}, \ldots, e^{t \mu_{m-1}(\varepsilon)}\right) \mathfrak{M}^{-1},
$$

где $\mu_{m}(\varepsilon)=-f^{(1)}-\varepsilon f^{(2)}-\varepsilon^{2} \cdots$,

$$
\begin{gathered}
\mu_{j}(\varepsilon)=-\varepsilon^{(2-m) /(m-1)}\left(q_{1, j}+\varepsilon^{1 /(m-1)} \frac{\gamma_{3-m}}{(m-1) q_{1, j}^{m-3}}+\varepsilon^{2 /(m-1)} \ldots\right) \\
1 \leqslant j \leqslant m-1
\end{gathered}
$$

(cp. c (16.13)).

ЗАмечАниЕ 17.1. Если $\sigma_{1, m-1}=0$, то $\Omega_{1-m}=0$ и преобразование $I+\varepsilon \Omega_{1-m}$ преврашается в тождественное.

\section{$\S 18$. Сильное вырождение структурной матрицы. Случаи I), II) в подпространстве End $P E$}

18.1. Переход к системе независимых уравнений. Пусть теперь $2 \leqslant$ $m_{1} \leqslant(m-1) / 2$ в соотношениях (9.2). Обратимся к расщепленной системе (15.10) и заметим, что второе уравнение из $(15.10)$ в $\operatorname{End}(I-P) E$ с функциями $G^{(i)}(t)$ из (14.14) изучено в п. 16.1. Первое уравнение из (15.10) в End $P E$ с функциями $F^{(i)}(t)$ из (14.12) рассматривалось в п. 15.6. Оно по форме напоминает уравнение (15.5) в пространстве $\mathfrak{N}$, поэтому можно использовать алгоритм, изложенный в $\S 15$, и ввести в рассмотрение преобразование подобия

$$
\mathfrak{M}_{1}(t, \varepsilon)=\operatorname{diag}\left(\mathfrak{M}_{1}(t, \varepsilon) ; 0_{n}\right)
$$

типа (16.3), действуюшее в End $P E$.

По аналогии с предположением 8.1 и формулами $(8.2),(8.3)$ будем считать выполненным

ПрЕДПОЛОЖЕНИЕ 18.1. Справедливы неравенства $1 \leqslant s_{1} \leqslant m_{1}$, или $0 \leqslant$ $m_{2} \leqslant\left(m_{1}-1\right) / 2$.

Здесь используются обозначения $m_{1}=m_{2}+n_{1}, s_{1}=n_{1}-m_{2}$. Важно отметить, что исследование заканчивается, когда в End $P E$ имеет место случай I) (при $m_{2}=$ $0)$ или случай II) (при $m_{2}=1$ ). Если же в подпространстве End $P E$ возникает случай сильного вырождения (при $2 \leqslant m_{2} \leqslant\left(m_{1}-1\right) / 2$ ), то после первой редукции задачи следует повторить аналогичные рассуждения.

В согласии с формулами из п. 16.2 и $§ 17$ получаем, что в случае I)

$$
\begin{aligned}
W_{P}(t, \varepsilon)= & \mathfrak{M}(t, \varepsilon) P \mathfrak{M}_{1}(t, \varepsilon) \operatorname{diag}\left(e^{\widetilde{\mu}_{n+1}(t, \varepsilon)}, \ldots, e^{\widetilde{\mu}_{m}(t, \varepsilon)} ; 0_{n}\right) \\
& \times \mathfrak{M}_{1}^{-1}(0, \varepsilon) P \mathfrak{M}^{-1}(0, \varepsilon),
\end{aligned}
$$


а в случае II)

$$
\begin{aligned}
W_{P}(t, \varepsilon)= & \mathfrak{M}(t, \varepsilon) P \mathfrak{M}_{1}(t, \varepsilon) \operatorname{diag}\left(e^{\widetilde{\mu}_{m}(t, \varepsilon)} ; e^{\widetilde{\mu}_{n+1}(t, \varepsilon)}, \ldots, e^{\widetilde{\mu}_{m-1}(t, \varepsilon)} ; 0_{n}\right) \\
& \times \mathfrak{M}_{1}^{-1}(0, \varepsilon) P \mathfrak{M}^{-1}(0, \varepsilon) .
\end{aligned}
$$

И тогда в силу $(16.2),(18.2),(18.3)$ получаем

$$
W(t, \varepsilon)=W_{P}(t, \varepsilon)+W_{I-P}(t, \varepsilon) .
$$

Далее опишем структуру матрищы $\mathfrak{M}_{1}(t, \varepsilon)$ и укажем вид скалярных функций $\widetilde{\mu}_{j}(t, \varepsilon), n+1 \leqslant j \leqslant m$, в каждом из случаев I), II).

Предварительно отметим, что матрицы $F^{(i)}(t)$ порядка $m_{1} \times m_{1}$ действуют в End $P E$ и ведут свое происхож дение от матриц $H_{i+1-n}(t)$, имеющих от одной до трех ненулевых линий, параллельных диагонали (см. (8.1), (10.5), (11.5)). В 112 (см. п. 12.3) указано, что номера линий однодиагональных (или двухдиагональных, когда $\left.1 \leqslant s \leqslant m_{1}-2\right)$ матриц $F^{(i)}(t)$ при всех $1 \leqslant i \leqslant m+n-2$ могут изменяться от $1-m_{1}$ до $m_{1}-1$. Из лемм 12.6-12.8 вытекает, что ненулевые элементы однодиагональных матриц $F^{(1)}(t), \ldots, F^{(s-1)}(t)$ располагаются на линиях с неотрицательными номерами. Матрица $F^{(s)}(t)$ содержит один ненулевой элемент, стоящий в $m_{1}$-й строке и 1-м столбце, и является первой однодиагональной матрицей с отрицательным номером, причем наименьшим.

Поскольку матрица $F^{(i)}(t)$ по структуре совпадает с $H_{11}^{(i+1-n)}(t)$, то случай I) в End $P E$ может возникнуть только при $i=s, i=s+n, i=s+2 n$ (последний имеет место, когда $\left.1 \leqslant s \leqslant m_{1}-2\right)$, а случай II) в End $P E$ - только при $i=s+1$, $i=s+n+1, i=s+2 n+1$ (последний также имеет место, когда $1 \leqslant s \leqslant m_{1}-2$ ).

В настоящей работе ограничимся рассмотрением случаев $i=s, i=s+1$. Будем обозначать сумму элементов матрицы $F^{(i)}(t)$ через $\gamma_{i, 1}(t)$. Если матрица $F^{(i)}(t)$ является двух диагональной, то условимся, что $\gamma_{i, 1}(t)$ - это сумма элементов матрицы с меньшим номером (большим весом).

18.2. Случай I) в End $P E$. Пусть $m_{2}=0, n_{1}=m_{1}$. Случай I) в подпространстве End $P E$ характеризуется соотношением

$$
\gamma_{s, 1}(t) \neq 0, \quad 0 \leqslant t \leqslant T .
$$

Возьмем матрицу срезания $\bar{\Lambda}_{1}(\varepsilon)$ из $(10.3)$, матрицу Вандермонда $\mathscr{V}_{1}(t)$ порядка $m_{1} \times m_{1}$, матрищу $Z_{1}(t, \varepsilon)$ с нулевой диагональю и положим

$$
\mathfrak{M}_{1}(t, \varepsilon)=\bar{\Lambda}_{1}(\varepsilon) \mathscr{V}_{1}(t)\left(I+\varepsilon^{\nu_{1}} Z_{1}(t, \varepsilon)\right) \text {. }
$$

Как показано в $[15], \bar{\nu}=s /\left(n m_{1}\right), \nu_{1}=\nu+\bar{\nu}=1 / m_{1}, \Gamma_{1}(t)=J_{1}-F^{(s)}(t)$. При выполнении условия (18.4) все ненулевые попарно различные собственные значения $q_{1}^{(1)}(t)$ матрицы $\Gamma_{1}(t)$ находятся из тождества

$$
\left(q_{1}^{(1)}(t)\right)^{m_{1}}+\gamma_{s, 1}(t) \equiv 0, \quad 0 \leqslant t \leqslant T .
$$

Так как $\mathfrak{M}^{-1}(0, \varepsilon)=O\left(\varepsilon^{(1-m) / n}\right), \mathfrak{M}_{1}^{-1}(0, \varepsilon)=O\left(\varepsilon^{s\left(1-m_{1}\right) / n m_{1}}\right)$, то

$$
P \mathfrak{M}_{1}^{-1}(0, \varepsilon) P \mathfrak{M}^{-1}(0, \varepsilon)=O\left(\varepsilon^{\left(1-2 m_{1}\right) / m_{1}}\right) \text {. }
$$

Следовательно, в (18.2) имеем

$$
\tilde{\mu}_{j}(t, \varepsilon)=\varepsilon^{-1} \int_{0}^{t}\left(\lambda(\tau)-\varepsilon^{1 / m_{1}} q_{1, j}^{(1)}(\tau)-\varepsilon^{2 / m_{1}} \ldots\right) d \tau, \quad n+1 \leqslant j \leqslant m .
$$


ТЕОРема 18.1. Пусть выполнены соотношения (9.2) при $2 \leqslant m_{1} \leqslant(m-1) / 2$ и справедливо предположение 15.1. Если, кроме того, $\gamma_{s, 1}(t) \neq 0$ при всех $0 \leqslant t \leqslant T$, то оператор Кочи уравнения (15.1) имеет вид

$$
\begin{aligned}
W(t, \varepsilon)= & W_{P}(t, \varepsilon)+W_{I-P}(t, \varepsilon) \\
= & \mathfrak{M}(t, \varepsilon)\left(P \mathfrak{M}_{1}(t, \varepsilon) \operatorname{diag}\left(e^{\widetilde{\mu}_{n+1}(t, \varepsilon)}, \ldots, e^{\widetilde{\mu}_{m}(t, \varepsilon)} ; 0_{n}\right) \mathfrak{M}_{1}^{-1}(0, \varepsilon) P\right. \\
& \left.\quad+\operatorname{diag}\left(0_{m_{1}} ; e^{\widetilde{\mu}_{1}(t, \varepsilon)}, \ldots, e^{\widetilde{\mu}_{n}(t, \varepsilon)}\right)\right) \mathfrak{M}^{-1}(0, \varepsilon)
\end{aligned}
$$

где матриць $\mathfrak{M}(t, \varepsilon), \mathfrak{M}_{1}(t, \varepsilon)$ определяются равенствами (16.3), (18.5) соответственно, а скалярные функиии $\widetilde{\mu}_{j}(t, \varepsilon)$ - равенствами (16.4), (18.8).

В [15] установлено, что

$$
\gamma_{s, 1}(t)=\frac{1}{\gamma_{1-n}(t)} \sum_{r=m_{1}}^{n} \sigma_{r, 1}(t) \sigma_{m, r+1}(t),
$$

поэтому имеет место

СлЕДСТВИЕ 18.1. Если в условиях предположения 15.1 выполнены соотношения (9.2) и, кроме того, $\sum_{r=m_{1}}^{n} \sigma_{r, 1}(t) \sigma_{m, r+1}(t) \neq 0$ при всех $0 \leqslant t \leqslant T$, то справедливо представление (18.9) оператора Кочи уравнения (15.1) с матрицами $\mathfrak{M}(t, \varepsilon), \mathfrak{M}_{1}(t, \varepsilon)$ из (16.3), (18.5) соответственно и скалярными функциями $\widetilde{\mu}_{j}(t, \varepsilon)$, определяемыми равенствами (16.4), (18.8).

18.3. Случай II) в End $P E$. Пусть $m_{2}=1, n_{1}=m_{1}-1$. Напомним, что через $\gamma_{s+1,1}(t)$ обозначена сумма двух элементов однодиагональной матрицы $F^{(s+1)}(t)=F_{s+1,1}^{(0)}(t)+\mathscr{D}_{s+1,1}(t)$ с номером диагонали, равньм $2-m_{1}$. Примем

$$
\mathfrak{M}_{1}(t, \varepsilon)=\left(I+\varepsilon^{(s+1) \nu} \Omega_{-n_{1}, 1}(t)\right) \bar{\Lambda}_{1}(\varepsilon) \Phi^{(2)}(t)\left(I+\varepsilon^{\nu_{1}} Y_{1}(t, \varepsilon)\right)\left(I+\varepsilon^{\nu_{1}} Z_{1}(t, \varepsilon)\right) .
$$

Здесь $\nu=1 / n, m_{2}=1$, матрица $\bar{\Lambda}_{1}(\varepsilon), \bar{\nu}>0$, определяется в $(10.3), \nu_{1}=\nu+\bar{\nu}$, матрицы $\Omega_{-n_{1}, 1}(t)$ (однодиагональная), $\Phi^{(2)}(t), Y_{1}(t, \varepsilon)$ (сплетающая), $Z_{1}(t, \varepsilon)$ (с нулевой диагональю) аналогичны описанным выше.

Случай II) в подпространстве End $P E$ характеризуется соотношениями

$$
F^{(s)}(t) \equiv 0, \quad \gamma_{s+1,1}(t) \neq 0, \quad 0 \leqslant t \leqslant T
$$

Отдельно следует рассмотреть случай $m_{1}=2$, отличающийся от рассмотренного в п. 18.1 случая $m=2, m_{1}=0$ тем, что здесь $s=2>1$, но $s_{1}=$ $n_{1}-m_{2}=0<1$, и предположение 18.1 не вьполнено. Такое исследование было проведено в [15].

Пусть $3 \leqslant m_{1} \leqslant(m-1) / 2, m_{2}=1$. Тогда согласно [15] имеем $\nu_{1}=1 /\left(m_{1}-1\right)$, $\bar{\nu}=(s+1) /\left(n\left(m_{1}-1\right)\right), \Gamma_{1}(t)=J_{1}-\mathscr{D}_{2-m_{1}, 1}(t)$. При выполнении условия $(18.11)$ все попарно различные собственные значения $q_{1}^{(1)}(t)$ матрицы $\Gamma_{1}(t)$ находятся из тождества

$$
q_{1}^{(1)}(t)\left(\left(q_{1}^{(1)}(t)\right)^{m_{1}-1}+\gamma_{s+1,1}(t)\right) \equiv 0, \quad 0 \leqslant t \leqslant T .
$$


По аналогии с $\S 17$ находим, что

$$
\begin{gathered}
(I-P) \mathfrak{M}^{-1}(0, \varepsilon)=O\left(\varepsilon^{(1-m) / n}\right), \quad P \mathfrak{M}_{1}^{-1}(0, \varepsilon) P \mathfrak{M}^{-1}(0, \varepsilon)=O\left(\varepsilon^{-2}\right) \\
\widetilde{\mu}_{j}(t, \varepsilon)=\varepsilon^{-1} \int_{0}^{t}\left(\lambda(\tau)-\varepsilon^{1 /\left(m_{1}-1\right)} q_{1, j}^{(1)}(\tau)-\varepsilon^{2 /\left(m_{1}-1\right)} \cdots\right) d \tau \\
\quad n+1 \leqslant j \leqslant m-1 \\
\tilde{\mu}_{m}(t, \varepsilon)=-\int_{0}^{t}\left(q_{1, m}(\tau)+\varepsilon q_{2, m}(\tau)+\varepsilon^{2} \cdots\right) d \tau
\end{gathered}
$$

ТЕорема 18.2. Пусть выполнены соотношения (9.2) при $3 \leqslant m_{1} \leqslant(m-1) / 2$ и справедливо предположсение 15.1. Если, кроме того, $\gamma_{s, 1}(t) \equiv 0, \gamma_{s+1,1}(t) \neq 0$ при всех $0 \leqslant t \leqslant T$, то оператор Коши уравнения (15.1) имеет вид

$$
\begin{aligned}
W(t, \varepsilon)= & W_{P}(t, \varepsilon)+W_{I-P}(t, \varepsilon) \\
= & \mathfrak{M}(t, \varepsilon)\left(P \mathfrak{M}_{1}(t, \varepsilon) \operatorname{diag}\left(e^{\widetilde{\mu}_{m}(t, \varepsilon)} ; e^{\widetilde{\mu}_{n+1}(t, \varepsilon)}, \ldots, e^{\widetilde{\mu}_{m-1}(t, \varepsilon)} ; 0_{n}\right) \mathfrak{M}_{1}^{-1}(0, \varepsilon) P\right. \\
& \left.\quad+\operatorname{diag}\left(0_{m_{1}} ; e^{\widetilde{\mu}_{1}(t, \varepsilon)}, \ldots, e^{\widetilde{\mu}_{n}(t, \varepsilon)}\right)\right) \mathfrak{M}^{-1}(0, \varepsilon),
\end{aligned}
$$

где матрицы $\mathfrak{M}(t, \varepsilon), \mathfrak{M}_{1}(t, \varepsilon)$ определяются равенствами (16.3), (18.10) соответственно, а скалярнье функции $\widetilde{\mu}_{j}(t, \varepsilon)$ - равенствами (16.4), (18.13).

В [15] показано, что

$\gamma_{s+1,1}(t)=\frac{1}{\gamma_{1-n}(t)} \sum_{r=m_{1}-1}^{n}\left(\sigma_{r, 1}(t) \sigma_{m-1, r+1}(t)+\left(\sigma_{r, 1}(t)+\sigma_{r+1,2}(t)\right) \sigma_{m, r+2}(t)\right)$.

Таким образом, имеет место

СЛЕДСТВИЕ 18.2. Если в условиях предположения 15.1 выполнены соотношения (9.2) при $3 \leqslant m_{1} \leqslant(m-1) / 2$ и, кроме того,

$$
\begin{gathered}
\sum_{r=m_{1}}^{n} \sigma_{r, 1}(t) \sigma_{m, r+1}(t) \equiv 0 \\
\sum_{r=m_{1}-1}^{n}\left(\sigma_{r, 1}(t) \sigma_{m-1, r+1}(t)+\left(\sigma_{r, 1}(t)+\sigma_{r+1,2}(t)\right) \sigma_{m, r+2}(t)\right) \neq 0
\end{gathered}
$$

при всех $0 \leqslant t \leqslant T$, то справедливо представление (18.14) оператора Коши уравнения (15.1) с матрицами $\mathfrak{M}(t, \varepsilon), \mathfrak{M}_{1}(t, \varepsilon)$ из (16.3), (18.10) coответственно и скалярными функциями $\widetilde{\mu}_{j}(t, \varepsilon)$, определяемыми равенствами (16.4), (18.13). 


\section{Список литературы}

1. Panonopm И. М. О некоторых асимптотических методах в теории дифференциальных уравнений. Киев: Изд-во АН УССР, 1954.

2. Федорюк М. В. Асимптотические методы для линейных обыкновенных дифференциальных уравнений. М.: Наука, 1983.

3. Федорюк M. В. Асимптотические методы в анализе // Совр. проблемы матем. Фундамент. направления. Т. 13. М.: ВИНИТИ, 1986. С. 93-210.

4. Ломов С. А. Введение в общую теорию сингулярных возмущений. М.: Наука, 1981.

5. Коняев Ю. А. Теория возмушений в прикладных задачах. М.: Изд-во МЭИ, 1990.

6. Фещенко С.Ф., Шкиль Н.И., Николенко Л.Д. Асимптотические методы в теории линейных дифференциальных уравнений. Киев: Наукова думка, 1966.

7. Моисеев H. Н. Асимптотические методы нелинейной механики. М.: Наука, 1969.

8. Сотниченко Н. А., Фещенко C. Ф. Асимптотическое интегрирование дифференциальных уравнений // Препринт № 80.3. Киев: ИМ АН УССР, 1980.

9. Елисеев А.Г. Теория сингулярных возмушений для систем диффееренциальных уравнений в случае кратного спектра предельного оператора. I, II // Изв. АН СССР. Сер. матем. 1984. Т. 48. № 5. С. 999-1020; 1984. Т. 48. № 6. С. 1171-1195.

10. Елисеев А.Г., Ломов С.А. Асимптотическое интегрирование сингулярно возмущеннных задач // УМН. 1988. Т. 43. №3. С. 3-53.

11. Шкиль Н.И., Старун И.И., Яћовец В. П. Асимптотическое интегрирование линейных систем обыкновенных дифференциальных уравнений. Киев: Вища школа, 1989.

12. Вазов B. Асимптотические разложения решений обыкновенных дифференциальных уравнений. М.: Наука, 1968.

13. Абгарян K. А. Матричные и асимптотические методы в теории линейных систем. М.: Наука, 1973.

14. Чернышов К. И. Метод стандартного расщепления сингулярно возмущенных дифференциальных уравнений // Докл. АН СССР. 1990. Т. 311. №6. С. 1311-1316.

15. Чернышов K. И. Асимптотический анализ уравнения с фредгольмовым оператором при производной // Дис. ... докт. физ.-матем. наук. Киев: ИМ НАН Украины, 1992.

16. Чернышов К.И. Асимптотические разложения решения задачи Коши для одного линейного дифференциального уравнения // Дифференц. уравнения. 1992. Т. 28. №5. C. $765-779$.

17. Чернышов K. И. Об экспоненте матричного пучка, зависящего от параметра, в случае кратного спектра предельной матрицы // Spectral and evolution problems. V. 13. Proc. of the Thirteenth Crimean Autumn Math. School-Symposium (18-29 сентября 2003 г., Симферополь, Украина). Симферополь: Таврич. нац. ун-т, 2003. Р. 65-81.

18. Колесов Ю. С., Кубъикин Е. П. Минималњный алгоритм исследования устойчивости линейных систем // Исследования по устойчивости в теории колебаний. Ярославль: Изд-во ЯрГУ, 1977. С. 142-155.

19. Арнольд В.И. О матрицах, зависящих от параметров // УМН. 1971. Т. 25. № 2 . C. $101-114$.

20. Богаевский B. H., Повзнер A.Я. Алгебраические методы в нелинейной теории возмущений. М.: Наука, 1987.

21. Далецкий Ю. Л., Крейн М.Г. Устойчивость решений дифференциальных уравнений в банаховом пространстве. М.: Наука, 1970.

22. Крейн С. Г. Линейные дифференциальные уравнения в банаховом пространстве. М.: Наука, 1967.

23. Ахиезер Н. И., Глазман И. М. Теория линейнњх операторов в гильертовом пространстве. М.: Наука, 1966.

24. Территин $X$. Л. Асимптотическое разложение решений систем обыкновенных линейных дифференциальных уравнений, содержащих параметр // Математика. Сб. пер. 1957. T. 1. № 2. C. 29-59.

Воронежская государственная лесотехническая академия

E-mail: chern@vsau.ru
Поступила в редакцию 27.05 .2003 и 12.11 .2004 